


\section{Le Néolithique du Nord de la France dans son contexte européen : habitat et économie aux $4^{\mathrm{e}}$ et $3^{\mathrm{e}}$ millénaires avant notre ère}

\section{Actes $d u 29^{e}$ colloque interrégional sur le Néolithique Villeneuve-d'Ascq 2-3 octobre 2009}

Textes réunis par

Françoise BOSTYN

Emmanuelle MARTIAL

Ivan PRAUD 


\title{
LE NÉOLITHIQUE MOYEN II DANS LE SUD-OUEST DU BASSIN DE L'ESCAUT : NOUVEAUX ÉLÉMENTS DANS LE GROUPE DE SPIERE
}

\author{
Françoise BOSTYN, Cécile MONCHABLON, Ivan PRAUD \& Bart VANMONTFORT
}

\section{INTRODUCTION}

La tenue du colloque Internéo dans le Nord de la France nous offrait l'opportunité de faire un bilan des avancées concernant le Néolithique moyen II dans la région. En effet, après une période de calme dans les découvertes de sites néolithiques, le début des années 2000, grâce à une multiplication des opérations de fouilles préventives dans les départements du Nord et du Pas-de-Calais, a vu le nombre de sites néolithiques fortement augmenter avec en particulier la découverte de fossés d'enceinte à Lauwin-Planque (BOSTYN et al. 2006) et à Carvin (Monchablon et al. ce volume). Ainsi, les corpus de mobilier en particulier céramique et lithique (la faune étant malheureusement toujours mal conservée dans les milieux limoneux de la région) se sont considérablement étoffés (fig. 1). Ces apports permettent de réviser la situation régionale, de préciser le cadre chronologique des occupations et de compléter les comparaisons culturelles avec les régions limitrophes en particulier avec la Belgique, autour des questionnements relatifs aux influences entre les deux grands ensembles culturels Chasséen et Michelsberg au sein du groupe de Spiere récemment individualisé (VANMONTFORT 2001).

\section{HISTORIQUE DES RECHERCHES ET PROBLÉMATIQUES}

Dans le Nord de la France, la deuxième moitié du $\mathrm{V}^{\mathrm{e}}$ millénaire est marquée par la fin des influences danubiennes vers 4850 avant J.-C. et par un vide de quelques siècles, toujours non comblé à ce jour. À partirde 4300 avantJ.-C., dans une phase detransition entre l'Atlantique et le Subboréal, de nouvelles cultures se développent : le Michelsberg dans la vallée de l'Aisne et l'est de la France, en Belgique et en Allemagne, et le Chasséen, venu du sud-est de la France et qui influence largement l'ouest du Bassin parisien. Ces deux entités culturelles ne sont pas monolithiques et des différences régionales ont très vite été suggérées par I. Scollar pour le Michelsberg (SCOLlaR 1959). Par ailleurs, très tôt des influences réciproques ont été évoquées pour expliquer la présence dans chaque entité culturelle d'objets de traditions extérieures (comme au sein de la catégorie des armatures de flèches ou de certains types de vases) et le terme Chasséo-Michelsberg a été employé pour nommer ce phénomène d'influences réciproques (par exemple Prevost 1962). Dès 1959, Scollar évoquait la possibilité que le Chasséen forme le substrat du Michelsberg belge (SCOLLAR 1959). En 1976 et 1980 Louwe Kooijmans avait aussi noté la présence d'influences chasséennes en contexte du Michelsberg de Belgique (Louwe Kooijmans 1976, 1980).

Les premières recherches ont été orientées vers la constitution d'un cadre chronologique à l'image des travaux de J. Lüning et de J. Lichardus pour le Michelsberg et les cultures rhénanes contemporaines (LÜNING 1968 ; LICHARDUS 1976). La périodisation du Chasséen septentrional a été proposée en particulier à partir des travaux sur les sites fossoyés de hauteur de Jonquières, Catenoy et Boury-enVexin (Blanchet \& Martinez 1980). Les entités culturelles sont construites essentiellement sur la panoplie céramique de chacune, et différenciées sur la base de variations typologiques importantes, les différences mineures étant interprétées comme de possibles variantes intra-culturelles. Ainsi, les jeux de classements typo-chronologiques se sont orientés vers des débats sur l'origine des entités culturelles ou stylistiques (Dubouloz 1998, JeunESSE 1998, JeUNESSE et al. 2004). Les derniers travaux ont ainsi mis en relief le rôle du Bassin parisien dans la genèseetle développement dela culture Michelsberg après une période couvrant le Néolithique ancien jusqu'à l'horizon Épi-Roessen où les influx culturels provenaient de l'est.

Dans le sud-ouest du bassin de l'Escaut qui englobe une partie des départements du Nord et du Pas-de-Calais ainsi que l'ouest de la Belgique, les années 1970 ont apporté des données nouvelles permettant d'affirmer les influences du monde rhénan sur les populations locales. En Belgique, la contribution des sites deSpiennes et de Boitsfort a été fondamentale dès le départ, mais la découverte des sites de Neufvilles (De Heinzelinetal.1977), Thieusies (VermeERSCH \& Walter 1978) et du Kemmelberg 


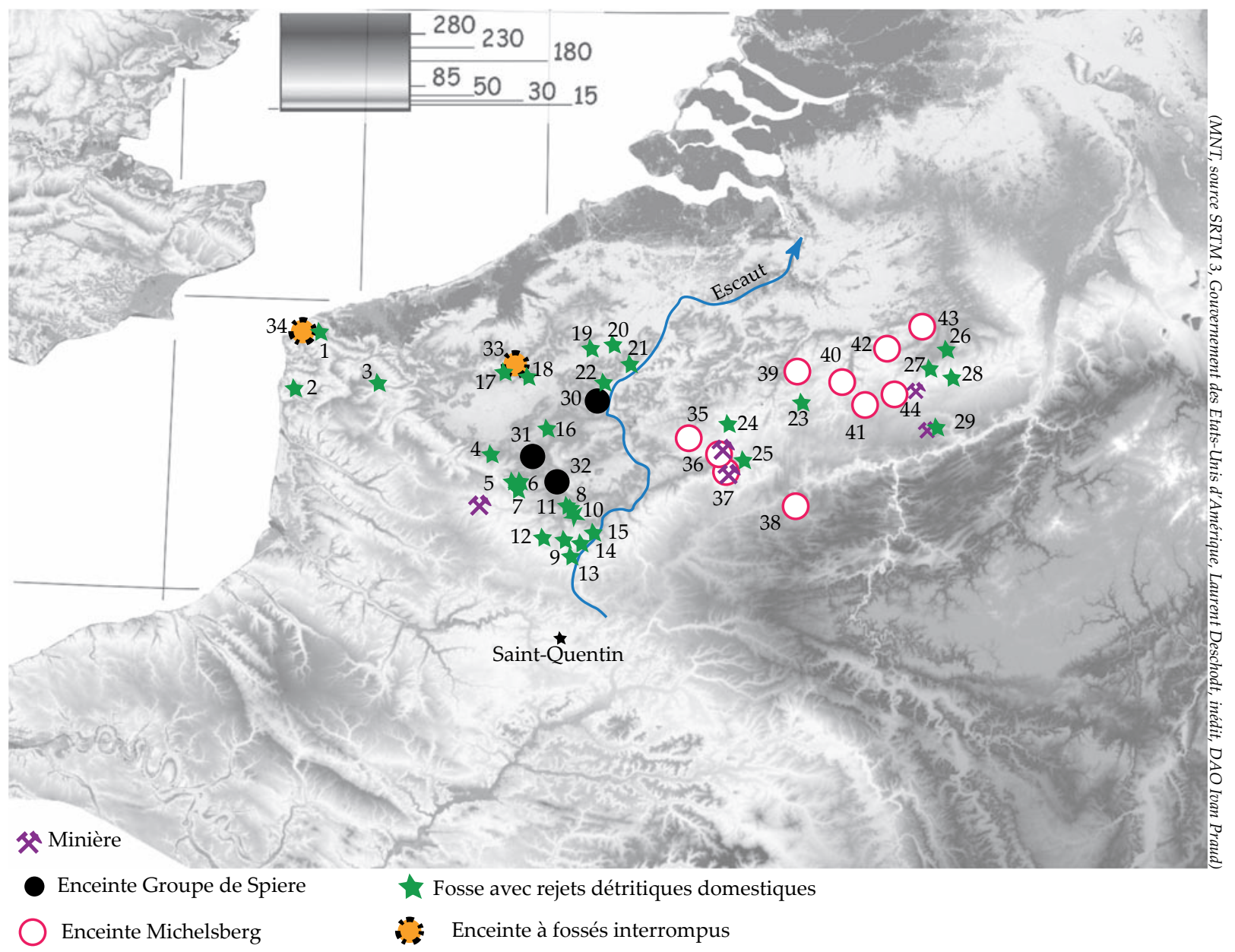

Fig. 1 - Répartition des sites dans l'aire d'influence du Groupe de Spiere et du Michelsberg Belge.

Site à fosse : 1- Coquelles ; 2- Isques ; 3- La Montagne de Lumbres ; 4- Sailly-Labourse ; 5- Liévin ; 6- Courrières ; 7- Avion ; 8- Brebières ; 9-Sauchy-Lestrée ; 10- Corbehem ; 11- Cherisy ; 12- Monchy-le-Preux ; 13- Masnières ; 14- Raillencourt-SainteOlle ; 15- Etrun ; 16- Houplin-Ancoisne ; 17- Le Mont Noir ; 18- Kooigem , 19- Petegem ; 20- Ename ; 21- Schorisse ; 22 Saint-Sauveur ; 23- Manage ; 24- Neufvilles ; 25- Spiennes ; 26- Wange ; 27- Orp-le-Grand ; 28 Avin ; 29 - Meeffe ; 45 - SauchyLestrée.

Les enceintes : 30- Spiere ; 31- Carvin "Gare d'Eau" ; 32- Lauwin-Planque "J. Cartier" ; 33- Kemmelberg ; 34- Escalles "Mont d'Hubert" ; 35- Blicquy ; 36- Thieusies ; 37- Petit-Spiennes 38- Thuin ; 39- Boistfort - Bosvoorde ; 40- Ottenburg - Grez Doiceau ; 41- Chaumont-Gistoux ; 42- Opvelp ; 43- Assent ; 44-Enines.

(VAN DoORDELAer 1971, VAN DOORDELAER et al. 1987) a complété de manière significative les assemblages céramiques. En parallèle, dans la région Nord-Pasde-Calais, aux premières découvertes faites sur le site de la Montagne de Lumbres (PREVOST 1962), se sont ajoutées des découvertes ponctuelles à Corbehem (Demolon \& Hurtrelle 1975), Etrun (Leman \& Leman 1973) et Sailly-Labourse (Leman 1978). Mais c'est surtout la découverte fortuite d'une fosse lors de travaux à Liévin en 1968 (TUFFrEAU 1970), puis une intervention sur une seconde fosse en 1976 par J.-F. Piningre qui ont livré des assemblages céramiques et lithiques plus importants permettant à ce dernier d'apporter des éléments nouveaux à la discussion (PININGRe 1985). Typologiquement, des influences du Michelsberg ancien et du Chasséen se trouvent rassemblées dans le mobilier céramique confirmant l'interpénétration importante des deux courants culturels bien au-delà de la Picardie. À la même époque, une première proposition de synchronisation des cadres chronologiques entre l'Alsace, l'Allemagne, le nord-est du Bassin parisien et la Belgique voyait le jour sur la base des comparaisons céramiques agrémentées des dates ${ }^{14} \mathrm{C}$ pourtant encore très imprécises à l'époque (JEUNESSE 1982).

Toutes ces idées et la découverte d'une enceinte à Spiere (Flandre, Belgique), dont une portion a pu être fouillée (VANMONTFORT et al. 1997), ont mené dès 2001 à la création d'une entité originale dans le sud-ouest du bassin de l'Escaut : «le groupe de Spiere ", groupe qui se trouve dans la zone d'interaction entre le Michelsberg et le Chasséen septentrional (VANMONTFORT 2001, 2004, 2006). L'identification de ce groupe s'est faite essentiellement sur des critères céramiques, qu'ils soient aussi bien d'ordre technologique, typologique que stylistique. Les autres matériaux sont aussi évoqués mais ne sont pas primordiaux dans la 
démonstration. Plusieurs critères (dégraissant, type de décor, formes) se sont révélés suffisamment différents des assemblages Michelsberg rhénan pour justifier la création de cette entité régionale, qui s'est développée tout en conservant des liens étroits avec les groupes régionaux voisins. Les caractéristiques identitaires de ce groupe ainsi que sa genèse au sein des processus de disparition du monde danubien et de mise en place des cultures du Néolithique moyen restaient cependant encore à conforter par de nouvelles découvertes afin aussi de mieux circonscrire son extension géographique en particulier vers l'ouest.

Le Nord de la France (départements du Nord, du Pas-de-Calais, mais aussi de la Somme) constituait de fait un laboratoire d'observation privilégié pour continuerà réfléchir sur ces problématiques, d'autant que la bordure orientale semblait déjà relativement bien fixée. Le développement de l'archéologie préventive dans ces régions a, durant les deux dernières décennies, apporté un nombre important de nouvelles données. Si la majorité des sites n'est représentée que par une ou deux fosses comme à Cherisy, Isques, Monchy-le-Preux, Courrières, ou encore Coquelles, des groupes de fosses ont pu être découverts sur des décapages plus importants, commeà Masnières ( 9 fosses de rejets) ou à Corbehem (12 fosses). Enfin, et ce fut une importante avancée pour la région qui n'en avait jamais livré, des sites à enceinte fossoyée ont été mis au jour à LauwinPlanque (Bostyn et al. 2006), à Carvin (MonchaBlon et al. ce volume) et dernièrement au Cap Blanc Nez à Escalles (WiLlot 2007), même si ce dernier doit plutôt être considéré comme un éperon barré. L'ensemble de ces sites n'est pas encore totalement étudié, mais les premiers résultats fournissent déjà matière à revenir sur plusieurs aspects de la culture matérielle et à rediscuter de l'entité culturelle du groupe de Spiere.

Nous nous proposons donc de reprendre l'ensemble des données archéologiques étudiées sur les gisements à fosse en les intégrant au sein des derniers bilans réalisés sur le groupe de Spiere (VANMONTFORT 2004).

Certaines de ces occupations ont fait l'objet d'une opération de fouille : Liévin (fosse 2 ; PININGRE 1985), Raillencourt-Sainte-Olle (BOSTYN et al. 1999), Avion (Feray 2003), Coquelles (Decheyer 2006) et Corbehem "Rue de Gouy" (BOSTYN et al. 2009). D'autres ont été repérées de façon fortuite à l'occasion de fouilles mises en œuvre pour d'autres périodes chronologiques (antique ou Moyen Âge) : Monchyle-Preux (JACQUES et al. 1995) et Brébières (LEMAN 1982) ou à l'occasion de travaux comme à Liévin (fosse 1 ; TUFFrEAu 1970). Enfin, plusieurs structures n'ont été reconnues et fouillées que dans le cadre de diagnostics d'archéologie préventive : Masnières "La Couture" (Bostyn et al. 2006), Cherisy (DEBIAK \& DeBS 1996), Courrières (LANTOINE et al. 2006) et SauchyLestrée (Gaillard \& Gustiaux 2009).
Géographiquement, les récentes découvertes se situent majoritairement en rive gauche de l'Escaut sur la frange septentrionale de l'Artois, dans un contexte géologique dominé par des formations crétacées (fig 1). Seuls les sites de Coquelles, d'Isques et d'Escalles "Le Mont d'Hubert" ont été découverts sur la frange littorale du département du Pas-de-Calais.

\section{LA CÉRAMIQUE}

De ces gisements récemment fouillés, seuls les sites à fosses livrent des séries céramique étudiées complètement, ce qui n'est pas encore le cas des corpus des sites d'enceinte (BOSTYN et al. 2006, Manceau ce volume, Monchablon et al. ce volume). Ainsi, le mobilier céramique pris en compte provient principalement des sites à fosses et donc seulement de contexte détritique. Nous avons réuni les assemblages anciens et récents des gisements à fosses du Néolithique Moyen II régional pour permettre d'atteindre un corpus suffisant qui regroupe 57 individus complets ou semi-complets. Ce décompte n'intègre pas les «individu-vase » reconnus à partir d'un fragment de bord, de fond ou de décors, ni les autres types de production comme les disques en terre cuite.

La moitié de ce corpus provient de deux sites : Liévin (21\%) et Corbehem "Rue de Gouy" (26\%) ; pour le reste, les occupations livrent un nombre de vases qui varie de 1 à 5 individus par gisement.

L'emploi du silex chauffé et pilé comme dégraissant principal est récurrent dans les productions céramiques régionales. Sur certains vases, il peut être associé à des grains de chamotte ou à des végétaux, comme à Liévin, fosse 2 (Piningre op. cit.). À Corbehem "Rue de Gouy", la situation est aussi dominée par l'emploi de blocs siliceux éclatés au feu et concassés. L'utilisation de la chamotte est le plus souvent anecdotique tandis que l'ajout de végétaux est quasiment systématique. L'identification de ces végétaux est difficile sauf dans deux cas, où l'emploi complémentaire de mousse a été reconnu.

La disponibilité des matériaux siliceux dans la région semble un argument de poids dans les choix des potiers. De fait, les différentes entités culturelles du Néolithique moyen II dans le Nord-Ouest de l'Europe utilisent, comme dégraissant principal, des matériaux locaux tels que le calcaire, la coquille, le silex ou les matériaux métamorphiques au gré des potentialités géologiques de chaque région (voir VANMONTFORT 2004, p. 334). À ce dégraissant minéral dominant s'ajoute un dégraissant végétal qui a pu être identifié dans plusieurs ensembles comme une mousse (Neckera crispa) (Dubouloz 1988, ConstantiN \& KuIJPER 2002). Cette association de dégraissants est présente dès le Cerny, et surtout dans l'horizon 

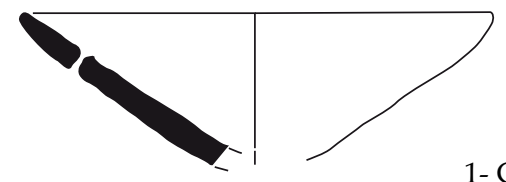

1- CORB27

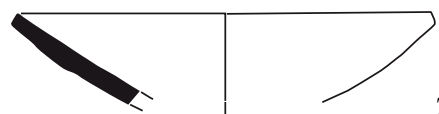

2- CORB27

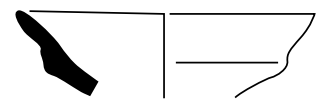

3- RSO 55

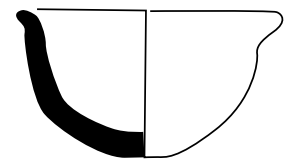

4- LUMB

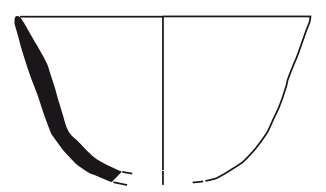

5- CORB14

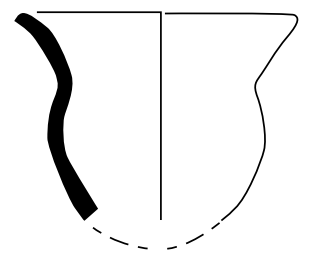

6- RSO 55

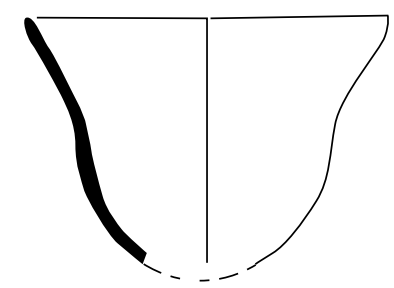

7- RSO 55

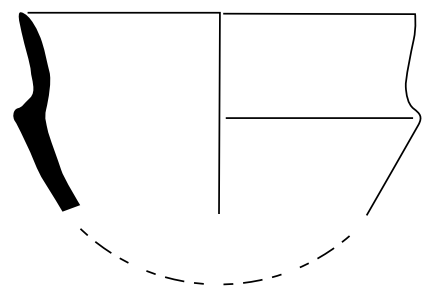

8- RSO 55

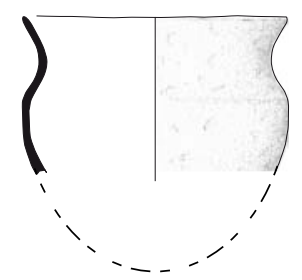

9- MLC St 15

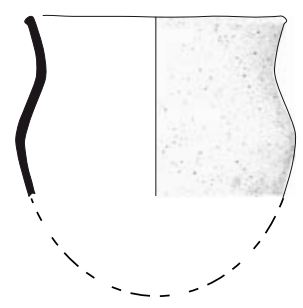

10- MLC st 15

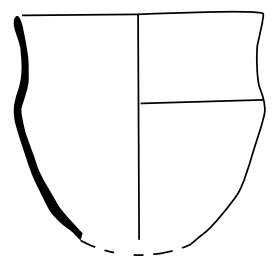

11- CHE

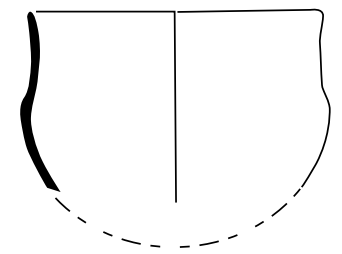

12- COQDIAG
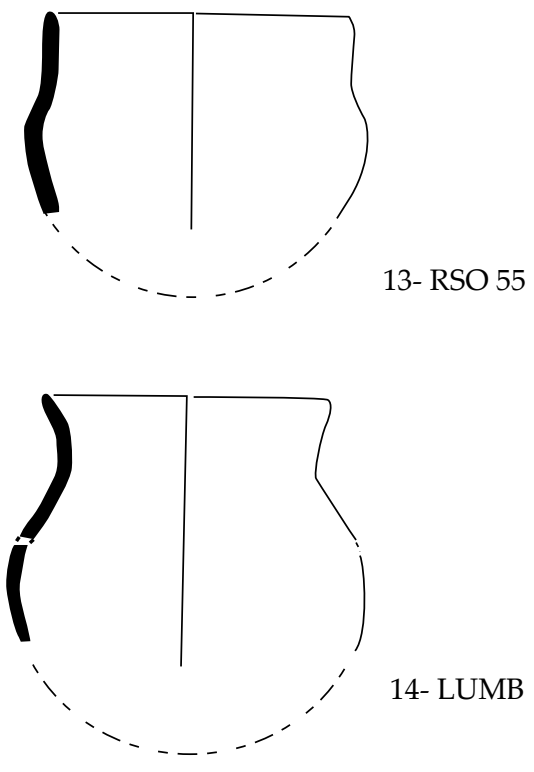

14- LUMB

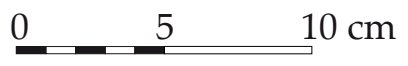

Fig. 2 - Formes basses et gobelets de petites dimensions des sites à fosses du Néolithique Moyen II du Nord - Pas-de-Calais ( $n^{\circ} 3,6,7,8$ et 13, d'après BOSTYN $1999 ; n^{\circ} 4$ et 14, PININGRE $1980 ; n^{\circ} 12$, DeCHEYER $2006 ; n^{\circ} 11$, DeBIAK E DeBs $1996 ; n^{\circ} 9$ et 10 GAILLARD $2006 ; n^{\circ} 1,2$ et 5, BOSTYN et al. 2009). Liste des abréviations : CHE = Cherisy ; COQ DIAG = Coquelles diagnostic ; $\mathrm{CORB}=$ Corbehem $; \mathrm{HA}=$ Houplin-Ancoisne $; \mathrm{ISQ}=$ Isques $; \mathrm{LIE}=$ Liévin $; \mathrm{LUMB}=$ Lumbres ; MLC = Masnières "La Couture" ; RSO = Raillencourt-Sainte-Olle ; SL = Sauchy-Lestrée 
Épi-Roessen/Bischheim occidental. La signification culturelle de ce choix technique a déjà été évoquée ailleurs (CONSTANTIN \& KUIJPER 2002). Au sein du groupe de Spiere, la mousse est utilisée de manière importante dans les assemblages céramiques en association avec le silex chauffé (VANMONTFORT 2005).

Les formes rencontrées se classent en cinq catégories principales : les formes basses simples et
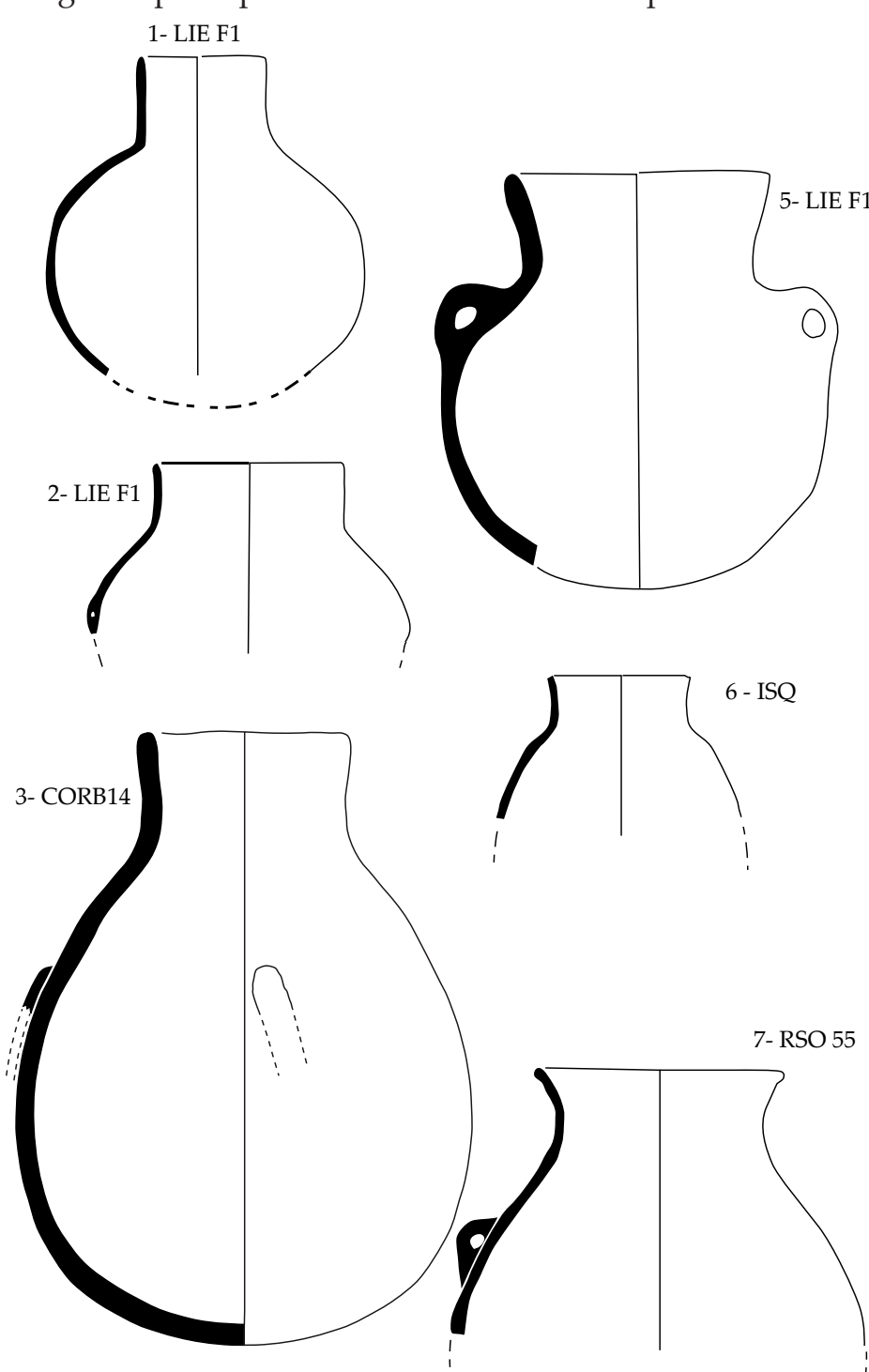

4- LIE F1

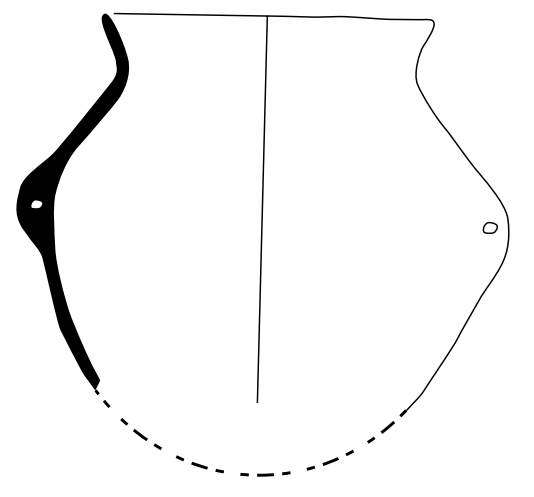

A

complexes (23\% ; fig. 2), les bouteilles / jarres à col souvent droit, parfois légèrement ouvert (17 \% ; fig. 3) et les vases à col oblique (35\% ; fig. 4 et 5).

Dans leur morphologie, les assemblages céramiques régionaux se caractérisent aussi par un nombre important de formes basses et de petits modules montrant parfois un profil à épaulement et un col oblique. C'est le cas notamment sur les sites

8- LIE C IIIb
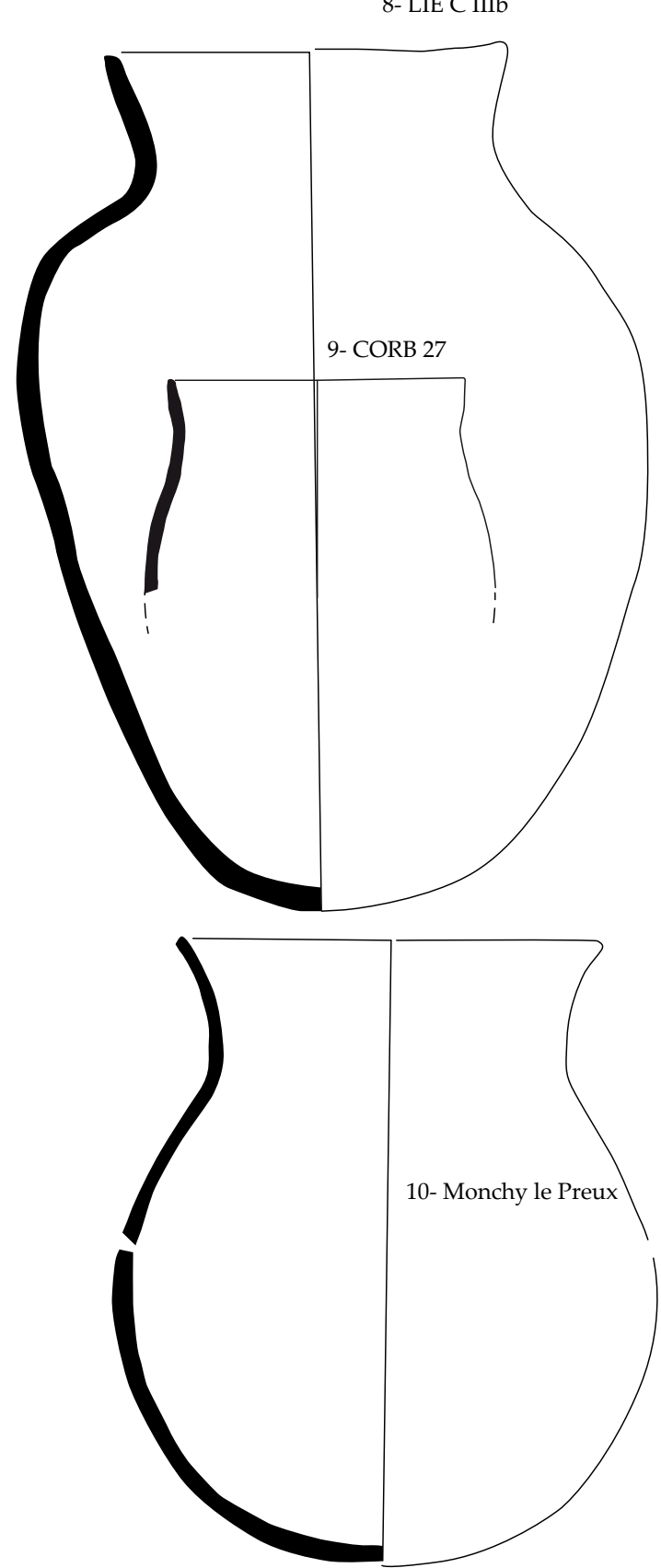

B

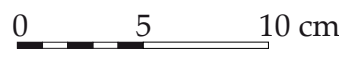

Fig. 3 - Bouteilles (A) et jarres (B) des sites à fosses du Néolithique moyen II régional $\left(n^{\circ} 1,2,4,5\right.$ et 8 d'après PININGRE 1985 ; n 6 Lepaumier 1996 ; n 7, BOSTYN 1999; n 10, Jaclues 1995, n 3 et 9 BOSTYN et al. 2009). 

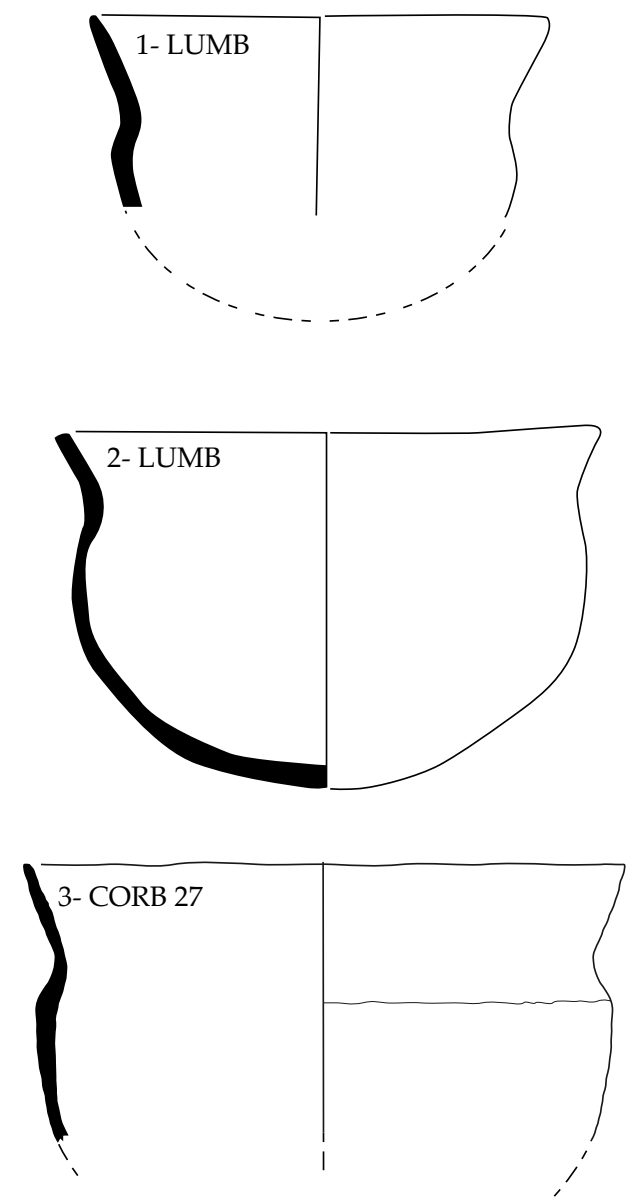
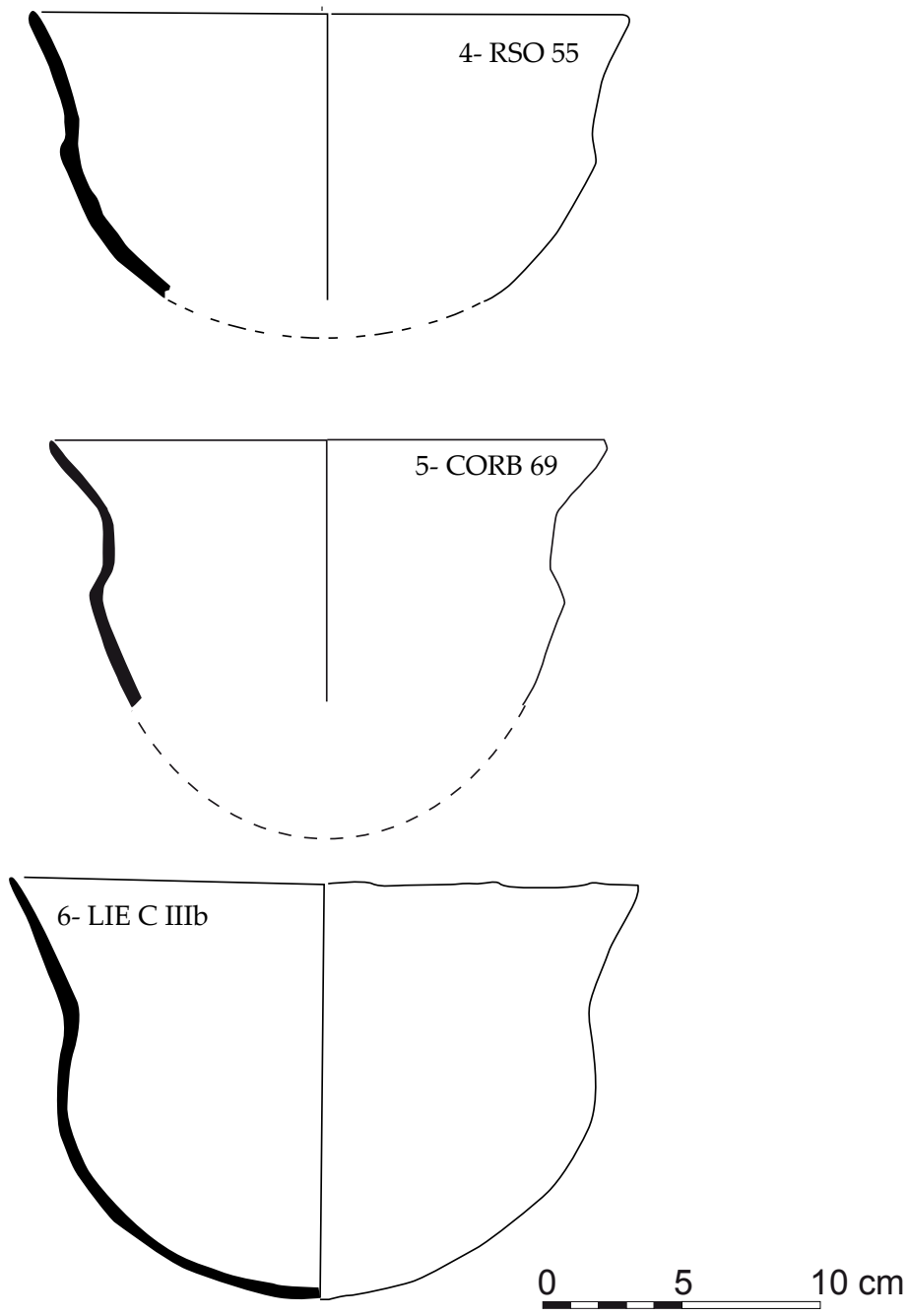

Fig. 4 - Vases à col ouvert du Néolithique moyen II régional $\left(n^{\circ} 1,2\right.$ d’après PININGRE $1980 ; n^{\circ} 6$ PININGRE $1985 ; n^{\circ} 4$ BOSTYN $1999 ; n^{\circ} 3$ et 5 BOSTYN et al. 2009).

de Raillencourt-Sainte-Olle et de Chérisy (fig. $2 \mathrm{n}^{\circ} 3$, 8 et 11). La présence de carène franche a été repérée sur des tessons fragmentés et qui sont souvent représentés dans les séries en un seul exemplaire comme à Corbehem "rue de Gouy".

Les moyens de préhension et de suspension sont plutôt rares mais variés. On trouve des anses doubles sur l'épaule à perforation horizontale (fig. $3, \mathrm{n}^{\circ}$ 5) et des mamelons perforés horizontalement à ensellement médian comme à Liévin fosse 1 (fig. $3, \mathrm{n}^{\circ} 2$ ), des boutons plus ou moins allongés non perforés et des languettes biforées à Houplin-Ancoisne (fig. 5, n ${ }^{\circ}$ ). Ces éléments sont toujours situés soit sur le haut de la panse pour les bouteilles ou placés à son diamètre maximum.

Les disques en terre cuite sont représentés sur un tiers des gisements archéologiques et les puisoirs sont rarissimes : il en existe un exemplaire sur le site de Raillencourt-Sainte-Olle "Le Grand Camp" (fig. 6, $\left.n^{\circ} 3\right)$.

Le registre des décors régionaux est pauvre comparé au nombre d'individus étudiables, ce qui correspond bien à ce qui est habituellement reconnu dans les séries du Néolithique moyen II. Le niveau archéologique de Sailly-Labourse, régulièrement mentionné dans les publications, est le seul gisement à avoir livré des motifs décoratifs variés tant du point de vue des techniques (poinçon, incision ou bouton au repoussé) que des thématiques : triangles hachurés, rameaux et bandes poinçonnées, décor de pastilles sur le bord interne d'un vase, lèvre encochée. D'autres vases présentent aussi des lèvres encochées ou décorées d'impressions obliques et de fines incisions (Corbehem "rue de Gouy" ; BOSTYN et al. 2009) ou encore des pastilles au repoussé sur le bord interne du vase (Corbehem "rue des Acacias"; Demolon \& Hurtrelle 1975).

Les sites d'enceinte complètent un peu ce corpus en y ajoutant des décors composés de pastilles au repoussé sur certains récipients et appliquées sur d'autres, mais toujours localisées sur la partie externe des vases et des impressions rectangulaires ou digitées comme à Lauwin-Planque. Ou encore en y apportant des éléments nouveaux comme ces incisions en chevron et ces impressions de coup 

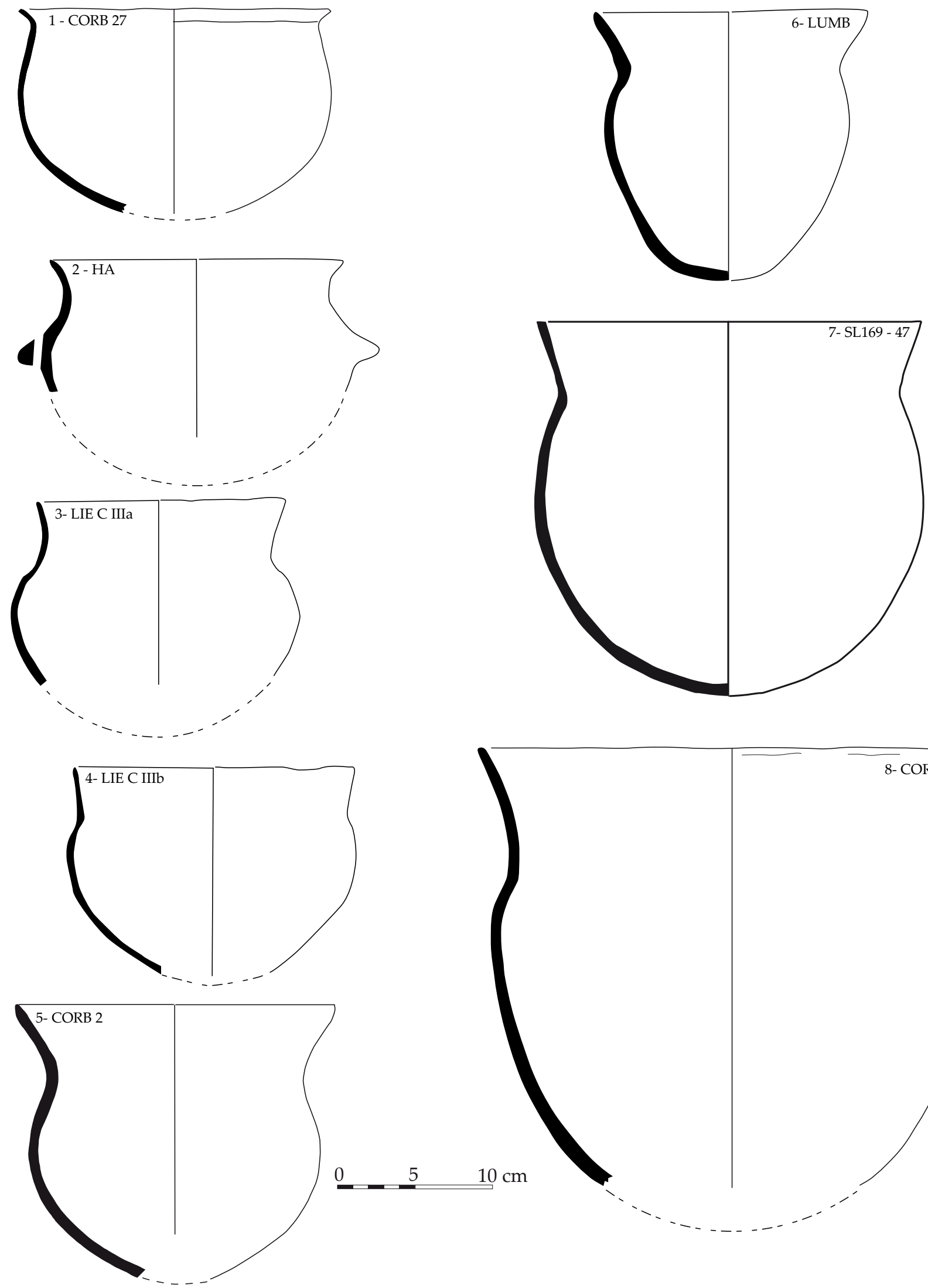

Fig. 5 : Marmite (1), vases à col du Néolithique moyen II régional $\left(n^{\circ} 2,3,4\right.$ et 6 d'après PININGRE $1985 ; n^{\circ} 7$ d'après GAILLARD $\mathcal{E}$ Gustiaux 2009; $n^{\circ} 1,5$ et 8 Bostrn et al. 2009). 

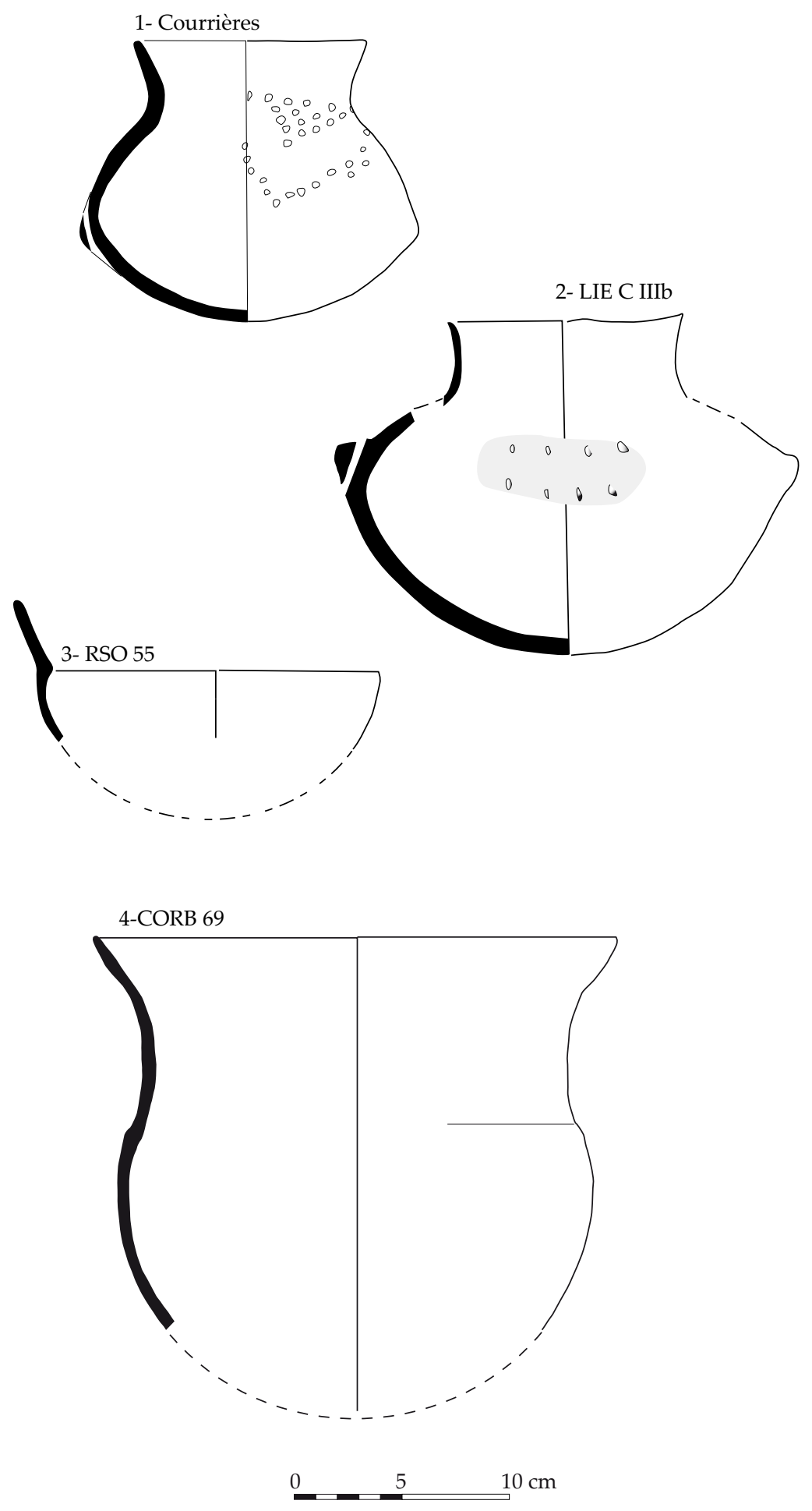

Fig. 6 - Céramique décorée et formes particulières des sites à fosses du Néolithique moyen II régional $\left(n^{\circ} 1 d^{\prime} a p r e ̀ s ~ L A N T O I N E\right.$, $2006 ; n^{\circ} 2$ PININGRE $1985 ; n^{\circ} 3$ BOSTYN $1999 ; n^{\circ} 4$ BOSTYN et al. 2009)

« d'aiguille » pratiquées à la jonction col/panse d'un gobelet sur le site de Carvin (Monchablon et al. ce volume).

Enfin, la présence au sein de ces collections de vases au profil particulier et souvent en un unique exemplaire permettrait d'identifier des éléments d'influence " extérieure ». Il s'agit ici de trois récipients provenant des sites de Courrières, Liévin et Corbehem "rue de Gouy".
Le premier, dégraissé au silex et à l'aide de fibres végétales (LANTOINE et al. 2006), se caractérise par une embouchure évasée, un col resserré et une panse ellipsoïdale comportant sur le diamètre maximum une perforation verticale sous-cutanée (fig. 6, n 1 ). Il présente un décor sur la partie supérieure caractérisé par une accumulation de coups de poinçon, profonds et circulaires, s'organisant en panneau triangulaire plus ou moins régulier à la jonction $\mathrm{col} /$ panse tandis qu'une bande simple descend en 
guirlande sur le haut de la panse. La forme du vase de Courrières n'est pas sans rappeler celle du vase de Liévin (fig. 6, $\mathrm{n}^{\circ} 2$ ) avec toutefois des nuances dans le diamètre maximum de la panse situé dans la partie inférieure du vase, dans le système de suspension constitué d'une perforation unique et dans la rupture marquée du profil évoquant les vases carénés. Cette forme rappelle, d'ailleurs, les petits vases à carène plus ou moins franche que l'on trouve dans les séries Michelsberg du nord-est du Bassin parisien (Bazoches-sur-Vesles, Dubouloz 1998 p. 11, fig. 1 ; Cuiry-les-Chaudardes, Le Bolloch 1984 p. 141, fig. 12) ou dans les ensembles du Chasséen ancien de la vallée de l'Oise (comme à Boury-enVexin, LOMBARDO et al. 1984 p. 278, fig. 10, $\mathrm{n}^{\circ}$ 3) mais qui ne sont, en revanche, jamais décorés. De la même manière, la technique du décor poinçonné trouve des parallèles dans le groupe de Spiere, exécuté ici sur la paroi externe d'un fragment de louche (VANMONTFORT 2004 p. 28 fig. 17, n 10) ou encore sur différents types de support en contexte Chasséen (Blanchet \& Burnez 1984).

Le second vase se remarque par une forme proche du précédent ; il présente un col droit, une panse ellipsoïdale et un système de suspension composé d'une languette multiforée localisée au diamètre maximum de la panse (fig. 6, $\mathrm{n}^{\circ} 2$ ). Les enceintes de Spiere "De Hel" et de Carvin "Gare d'Eau" offrent des spécimens comparables et à ce jour reconnus en un seul exemplaire par site. Là encore, sur la base uniquement de la présence de ces systèmes de préhension, les séries Michelsberg de la vallée de l'Aisne et chasséennes de la vallée de 1'Oise soutiennent la comparaison : comme à Maizy (Dubouloz et al. 1984, p. 119 fig. 5, n³) ou encore à Catenoy "Le Camp César" (Blanchet et al. 1984, p. 185, fig. 10).

Enfin, le dernier exemplaire se rapproche des formes possédant un col très développé $(9 \mathrm{~cm})$ et ouvert, souligné à la jonction col/ panse par un sillon créant un léger épaulement sur la panse (fig. 6, n 4). Il s'apparente aux vases du Michelsberg ancien et Roessen tardif à bord épaissi.

En conclusion, le mobilier céramique mis au jour récemment conforte l'appartenance des sites de la région au groupe de Spiere tant à partir des formes, des décors que des techniques de fabrication.

Les influences culturelles des groupes du Michelsberg et du Chasséen sont indiscutables. L'augmentation du nombre de vases à col oblique et court dans le corpus régional renforce des éléments de parenté avec les assemblages Michelsberg du nord-est du Bassin parisien. Ce sentiment est conforté par l'assemblage de la série de Corbehem "rue de Gouy" et en particulier la présence d'une marmite à bord éversé (fig. 5, n 1) qui trouve des parallèles avec les exemplaires connus à Bazoches-
sur-Vesle ou à Maizy ou plus à l'est sur les sites de Mairy dans les Ardennes ou de Mayen en Allemagne. Les éléments chasséens sont toujours présents mais discrets; ils se caractérisent par ces petits vases à barrette multiforée, par ces bouteilles à col droit munies d'anse perforée horizontalement, par des mamelons à ensellement médian ainsi que par la présence de vases «à carène » franche dont les profils ne sont pas reconstituables pour l'instant.

Les éléments de filiation Michelsberg semblent plutôt liés à un faciès ancien de cette culture tels qu'ils sont décrits dans la sériation de Lüning (1968). Ceci peut être interprété de manière régionale/stylistique (VANMONTFORT 2004) ou bien chronologique (CONSTANTIN \& DEMAREZ 2009).

En somme, les éléments occidentaux dominent le corpus céramique (dans l'étape de formation) du groupe de Spiere. Ceux-ci montrent l'importance que le groupe de Menneville/Bischheim occidental joue dans sa genèse, ainsi que le Michelsberg ancien etleChasséen septentrional. Des éléments orientaux, liés par exemple au Roessen tardif, restent toutefois limités à certains motifs poinçonnés présents dans des cas plutôt isolés (Sailly-Labourse, Courrières).

\section{DU CÔTÉ DE L'INDUSTRIE LITHIQUE}

En Belgique le matériel lithique des sites de Thieusies (VERMEERSCH et al. 1990) et de Spiere (VANMONTFORT et al. 2004), a été étudié de manière presque systématique ce qui a permis la mise en avant de quelques éléments de synthèse (VERMEERSCH \& Burnez-LANOTTE 1998, V ANMONTFORT et al. 2009). Par contre, les connaissances de l'économie lithique des sites d'habitat dans le Nord de France se résumaient, jusqu'à peu, essentiellement aux données issues de la fouille de Liévin (PININGRE 1985). En effet, ainsi que le rappelait J.-F. Piningre (op. cit. p. 437), l'autre série abondante provenant du site de Lumbres (PREVOST 1962) n'est pas à l'abri de mélanges du fait de la présence du niveau Cerny sous-jacent. Le corpus du site fossoyé de Carvin étant en cours d'analyse à ce jour, nous n'en tiendrons pas compte dans cette présentation. Celui de l'enceinte de Lauwin-Planque n'a été étudié que partiellement, mais les premières informations recueillies viennent utilement compléter cette approche (BOSTYN et al. 2006). Les quelques ensembles retenus pour cet article sont donc issus essentiellement de fosses qui ne sont pas intégrées dans une enceinte. Parmi les sites dernièrement découverts, il faut néanmoins signaler que certains d'entre eux ont livré essentiellement du mobilier céramique comme les fosses de Cherisy ou de Monchy-le-Preux ou encore le puits à eau de Raillencourt-Sainte-Olle. Le mobilier lithique issu des fosses de Courrières est par ailleurs très particulier car sans doute sélectionné (LANTOINE et al. 2006) et ne peut apporter des informations généralisables à l'ensemble de l'économie lithique 

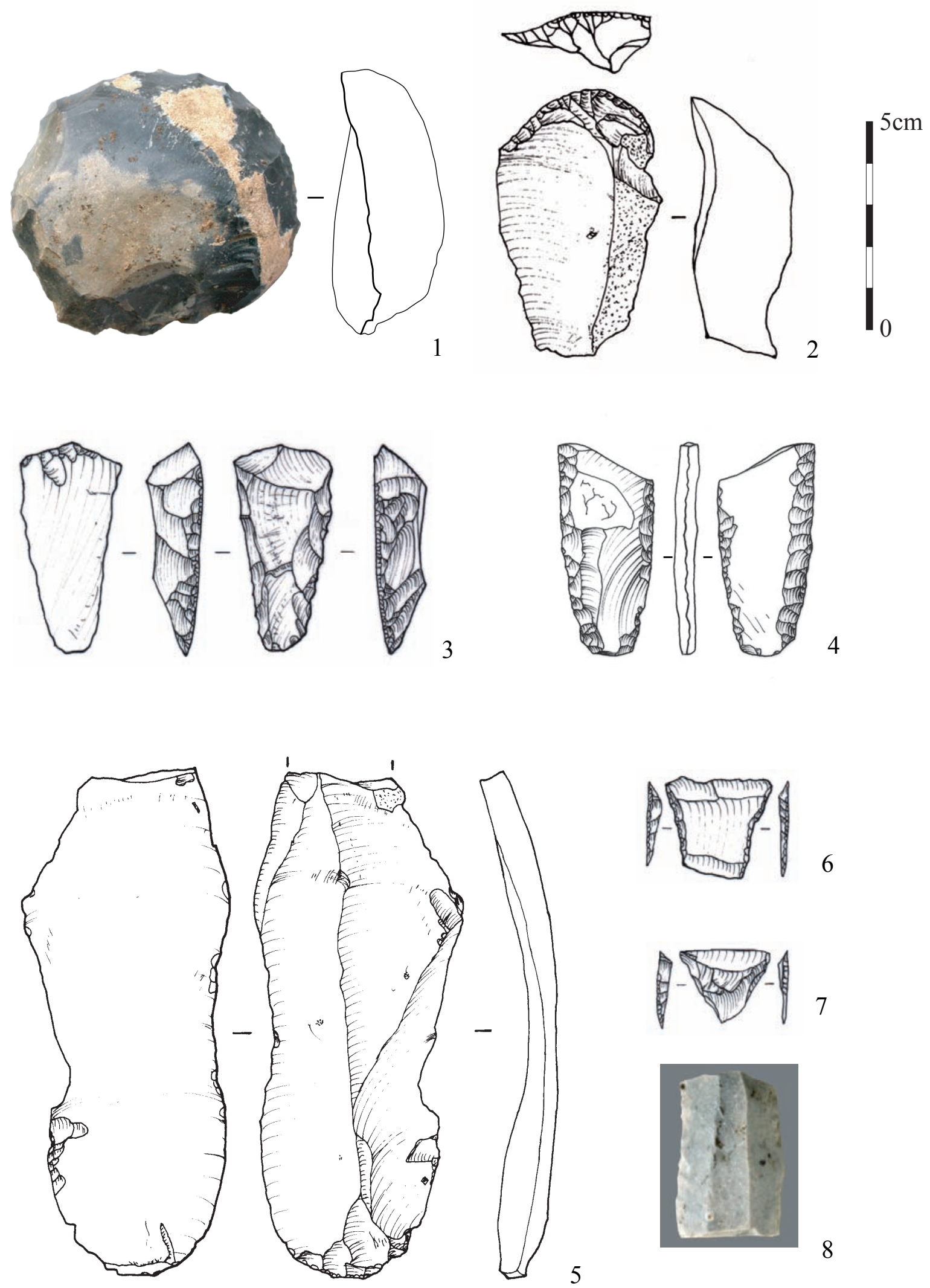

5

Fig. 7 - Industrie lithique des sites de Corbehem $\left(n^{\circ} 1,2,4\right.$ et 8$)$ et de Lauwin-Planque $\left(n^{\circ} 3,5,6\right.$, et 7). 1, 2-grattoirs ; 3-tranchet ; 4- armature foliacée ; 5, 8- lame ; 6, 7-armatures tranchantes. 1 et 8 : silex de Spiennes (dessins et photos F. BOSTYN). 
régionale. Ainsi la diversité des informations est nettement moindre que pour le mobilier céramique et les séries quantitativement représentatives peu nombreuses. Le site de Corbehem lui-même, fort de 779 artefacts, comporte un taux de pièces brûlées extrêmement élevé (plus de la moitié) qui constitue un handicap de taille pour une analyse fine (BOSTYN et al. 2009). Cette même observation avait déjà été faite à Liévin où un tiers des artefacts en moyenne a subi une chauffe.

Le recours à des matières premières locales apparaît comme une constante dans les assemblages lithiques étudiés. Il s'agit dans la région concernée des horizons du Crétacélivrant des rognons de forme globuleuse. Cependant, si à Liévin seule une hache était issue d'une roche dure exogène (dolérite), sur le site de Corbehem, des artefacts en silex issus de la minière de Spiennes ont été identifiés. Il s'agit de fragments de haches polies, d'un fragment de lame (fig. 7, $\mathrm{n}^{\circ}$ 8) et de deux grattoirs sur éclat (fig. 7, $\mathrm{n}^{\circ} 1$; BOsTYN et al. 2009). Au total, cette matière première représente 1,9\% de la série, mais probablement un peu plus puisque plus de la moitié des artefacts sont brûlés et non déterminés du point de vue de la matière première. Sur le site de Lauwin-Planque (Bostyn et al. 2006), le silex de Spiennes a fait l'objet également d'une importation mais il a été retrouvé exclusivement sous la forme de lames et de haches polies. Le rayonnement de la minière de Spiennes est donc sensible sur les sites du Nord - Pas-deCalais, même si on n'obtient pas des taux aussi élevés qu'à Spiere où ce silex représente environ $5 \%$ (VANMONTFORT et al. 2004), pour des distances comparables (environ $80 \mathrm{~km}$ ). L'importation de haches en dolérite depuis la Bretagne soit à plus de 700 km, notée à Liévin et au Kemmelberg (VAN DOORSELAER et al. 1987) est également probable à Lauwin-Planque même si la matière première des haches n'a pas été déterminée à ce jour.

Si les matières premières exogènes ont été recherchées pour l'approvisionnement des sites d'habitat en produits spécifiques (haches et lames) issus des grands centres miniers comme Spiennes, les matières premières locales ont été mises à profit pour l'obtention de supports pour la confection de l'outillage plus commun. Il s'agit d'une production d'éclats réalisée au percuteur de pierre selon des schémas opératoires simplifiés. Les éclats ont été sélectionnés en premier lieu pour la réalisation de grattoirs (fig. $7, \mathrm{n}^{\circ} 1,2$ ) qui représentent souvent près de la moitié de l'outillage $(49 \%$ à Liévin, $41 \%$ à Corbehem). Parmi les autres catégories d'outils, on signalera la présence de denticulés, d'éclats retouchés, de quelques perçoirs et racloirs. Les tranchets dont la présence est signalée sur les sites Michelsberg belges, sont présents à Lauwin-Planque (fig. $7, n^{\circ} 3$ ), mais absents à Liévin et Corbehem. Au sein de la catégorie des armatures de flèches, on a pu identifier aussi bien des armatures tranchantes (fig. 7, $\left.n^{\circ} 6,7\right)$ que des armatures foliacées (fig. 7, n 4), les deux types étant souvent associés. Une production de lame de petites dimensions a cependant aussi été faite sur place ainsi qu'en témoignent les quelques produits bruts ou retouchés étudiés à Corbehem et Lauwin-Planque. Cette production n'est cependant en rien comparable avec celle de Spiennes tant dans les dimensions (produits de moins de $10 \mathrm{~cm}$ ) que dans la qualité de la production (produits plus irréguliers). Néanmoins quelques rares produits sortent du lot suggérant des tentatives de produire des lames de meilleure facture (fig. 7, $n^{\circ} 5$ ). Aucun témoin pouvant se rapporter à une telle chaîne opératoire n'a cependant pu être isolé dans les séries lithiques. On observe des comportements proches au sujet des haches polies, puisqu'une partie des pièces est produite en silex local. Il s'agit peut-être ici de produits issus de minières locales comme celle de Fampoux (Masson \& VALLin 1994).

Les comparaisons avec le site de Spiere (VANMONTFORT et al. 2004) montrent de nombreuses ressemblances dans les comportements face à l'industrie en silex. On se réfère à la distinction entre une production spécialisée provenant de la minière de Spiennes et une production d'éclats sur les matériaux locaux. Concernant l'outillage, on notera une forte prédominance des grattoirs sur éclats et la présence de tranchets. Au sein de la catégorie des armatures de flèche, par contre, les armatures foliacées constituent le type nettement dominant.

Ces nouvelles données sur l'industrie lithique renforcent donc l'impression d'homogénéité de l'entité « groupe de Spiere » au sein de laquelle on perçoit l'impact important du site minier de Spiennes, tout en notant des différences par rapport aux industries lithiques Michelsberg, en particulier dans son faible caractère laminaire.

\section{L'INDUSTRIE MACROLITHIQUE ET SES APPORTS}

À l'heure actuelle, peu d'études ont été faites sur les industries macrolithiques de la sphère Michelsberg. Ce travail commence (MK Projekt, ANR- 09-FASHS-07) et l'esquisse proposée ici devra par la suite être complétée.

La région d'étude est largement couverte par les sédiments tertiaires du Thanétien dans lesquels on trouve des grès du Groupe de Landen (anciennement Landénien). Ils offrent différents degrés de cohésion qui témoignent de silicification différentielle au sein de cette formation, passant ainsi des grès tendres (peu cohérents) aux grès compacts (très homogènes) parfois dénommés «quartzite». Ces grès se présentent sous forme de bancs discontinus ou de blocs isolés au sommet de buttes résiduelles (PIRsON et al. 2001, 
fig. 4). Ces grès compacts forment l'essentiel des outils de mouture, broyage et polissage (PININGRE 1985, VermeERsch et al. 1990, Pirson et al. 2001, VANMONTFORT 2004, MONCHABLON dans BOsTYN et al. 2009), même si, plus rarement, des grès tendres ont également été sélectionnés. À Spiennes comme à Corbehem, un approvisionnement par ramassage de surface a été mis en évidence (PIRSON et al. 2001, Monchablon dans Bostyn et al. 2009).

S'il est prématuré de synthétiser les assemblages macrolithiques du Néolithique moyen II régional, quelques grandes lignes peuvent néanmoins être proposées.
Les activités de subsistance sont bien présentes et reconnues au travers de la mouture principalement. Mais les outils de mouture sont très souvent réduits à l'état de fragments (PININGRE et al. 1985, VERMEERSCH et al. 1990, PIRSON et al. 2001, VANMONTFORT 2004). Ainsi, actuellement, peu de sites ont livré des meules et molettes suffisamment complètes pour mériter descriptions et illustrations (fig. 8, n 1 à 3) (Thieusies, VermeERsch et al, 1990 ; Corbehem, Monchablon dans Bostyn et al. 2009). La plupart du temps, cette casse de l'outillage de mouture n'est pas le résultat d'accident, mais bien "d'un geste de fracturation volontaire, répété et codifié » (HAMON 2006, p. 143). La destruction des outils de

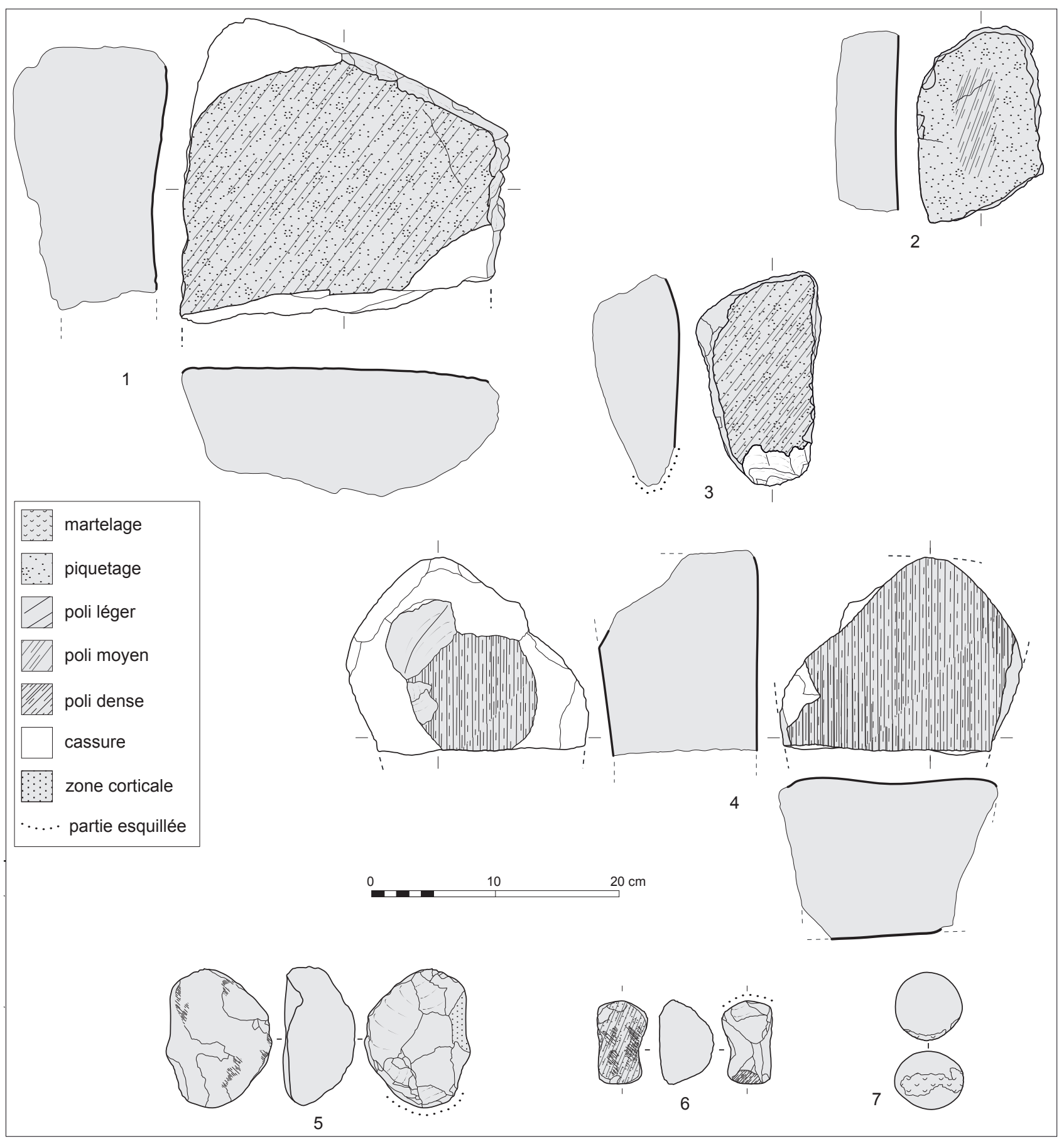

Fig. 8 - Outils en grès du site de Corbehem "rue de Gouy". 1 : meule ; 2 : molette ; 3 : molette-percuteur ; 4 : polissoir double face ; 5 : outil à plan de fracture émoussé et tranchant utilisé sur éclat; 6 : outil à plan de fracture émoussé et tranchant utilisé sur fragment thermique ; 7 : percuteur sur galet de silex roulé (DAO : Stéphane LANCELOT et Cécile MONCHABLON). 
broyage (avec ou sans réemploi des fragments) est un élément commun durant tout le Néolithique (Monchablon 1999, Pirson et al. 2001, Hamon 2006). Ce n'est donc pas tant la qualité de ces vestiges qui surprend que leur faible quantité (en NMI) par rapport aux autres activités.

En effet, les activités de transformation artisanale sont également très bien représentées. Elles semblent surtout tournées vers le polissage-abrasion et le raclage (travail de la peau, de végétaux...). Dans la panoplie des outils laissés sur les sites, on compte de nombreux polissoirs (fig. 8, $\mathrm{n}^{\circ} 4$ ) et outils à émoussé de raclage (fig. $8 \mathrm{n}^{\circ} 5$ et 6 ) et dans une moindre mesure des percuteurs (fig. $8, \mathrm{n}^{\circ} 7$ ) et des enclumes (PININGRe et al. 1985 ; VERMEERSCH et al. 1990 ; VANMONTFORT 2004 ; MONCHABLON dans Bostyn et al. 2009).

Cette forte présence des activités artisanales diffère des schémas connus pour le Néolithique ancien du Bassin parisien, du Hainaut et de la Hesbaye liégeoise et pour le Chasséen septentrional : la mouture y est plus largement documentée (HAMON 2006, Monchablon dans LANCHON et al. 1998, MonCHABLON dans GILIGNY dir. 2005). Faut-il comprendre cette relativement faible représentation des outils de mouture sur les sites du sud-ouest du Bassin de l'Escaut comme le reflet d'une production de subsistance plus tournée vers l'élevage que vers l'agriculture céréalière ? Il est pour l'heure difficile d'avancer plus loin dans cette hypothèse car la nature limoneuse des sites de la région ne permet pas une conservation des restes fauniques et altère par là les possibilités de dépeindre l'économie de subsistance principale (élevage bovin ou agriculture céréalière). En revanche, l'analyse plus systématique des macrorestes végétaux devrait apporter une part des informations quant au recul ou non des activités agricoles au Néolithique moyen II régional (DiETsCh-SELlAMi dans Bostyn et al. 2009).

En même temps que ce questionnement, il faudra répondre à la question du statut des sites désignés par une seule ou quelques fosses : site d'habitat, site de stockage, site à vocation artisanale ou autre?

\section{CHRONOLOGIE}

La question de la durée d'existence du groupe de Spiere est délicate, car les éléments nécessaires à une construction typo-chronologique des assemblages de mobilier manquent cruellement, comme les décors céramiques. Seule, l'évolution des formes semble exploitable pour voir une évolution de ce groupe (VANMONTFORT 2004). L'exercice n'est pas plus simple pour le Michelsberg du nord-est du Bassin parisien dont seules quelques étapes (les plus anciennes) seraient représentées (Dubouloz 1998).
Le recours à la méthode de datation ${ }^{14} \mathrm{C}$ peut se révéler inefficace dans le cadre d'une définition fine de l'évolution d'un groupe culturel si au préalable la discussion autour de la validité des résultats n'a pas été menée, au même titre que nous le faisons à partir des données archéologiques. Ce travail critique vise à conforter les données chronologiques existantes sur cette période afin de les intégrer à un projet européen plus vaste et qui concerne notamment la durée et les étapes constitutives du Michelsberg (MK Projekt, ANR- 09-FASHS-07).

\section{ANALYSE CRITIQUE DES MESURES RADIOCARBONE}

Les vingt-trois dates retenues dans cet article appartiennent à des sites attribuables au groupe de Spiere (tab. I et II). Une exception concerne le site de Kemmelberg (Flandre, Belgique) où l'insuffisance du mobilier ne permet pas une attribution fiable. Toutefois, sa position géographique milite en faveur d'une attribution au groupe de Spiere.

Les dates ont été obtenues à partir de charbons de bois (3/4) et de restes de fruits ou de céréales carbonisés (1/4). La moyenne générale de l'ensemble des datations situe le groupe de Spiere à la transition des $\mathrm{V}^{\mathrm{e}}$ et $\mathrm{IV}^{\mathrm{e}}$ millénaires avant notre ère, laissant croire qu'il pourrait y avoir un avant et un après 4000 ans (fig. 9). Mais l'étalement des dates sur un millénaire illustre ici les distorsions dues à un ensemble de phénomènes dans la calibration des résultats (fluctuation atmosphérique, marge statistique élevée, matériau daté...) que ne saurait masquer cette moyenne. À partir de données critiques objectives (issues des données physicochimiques et archéologiques), nous proposons de faire un tri sur nos propres résultats entre les dates qui sont susceptibles d'en diminuer la portée et celles « jugées » comme satisfaisantes. Ce travail critique avait déjà été en partie réalisé par l'un d'entre nous avec les dates disponibles à l'époque. Les huit mesures écartées sur des critères identiques n'ont, bien entendu, pas été reprises totalement dans cet article mais sont mentionnées uniquement dans le tableau II (tab. II, V ANMONTFORT 2004).

Sur la période, deux phénomènes dits de " plateaux » altèrent la courbe de calibration; ils ont pour principal effet d'étaler les mesures radiocarbones en années réelles sur des intervalles de temps répartis sur plusieurs siècles. Ils se situent entre la fin du $\mathrm{V}^{\mathrm{e}}$ et les débuts du IV millénaires, ce qui donne en valeur absolue une première fourchette de 5370 à $5240 \mathrm{BP}$, située en années réelles entre 4260 et 4080 . Le second plateau se trouve sur l'intervalle de 5100 à $5000 \mathrm{BP}$ soit un espace temps compris entre 3950 et 3790 ans (VANMONTFORT 2004). Sur les quinze dates (tab. I), huit se situent à l'intérieur de l'un de ces deux phénomènes, deux sont antérieures et cinq se situent entre les deux (fig. 9). 


\begin{tabular}{|l|l|r|r|l|}
\hline \multicolumn{1}{|c|}{ site } & Lab number & \multicolumn{1}{l|}{ date BP } & \multicolumn{1}{l|}{ stdev } & Material \\
\hline Kemmelberg & Lv-525 & 5020 & 95 & Charcoal \\
\hline Corbehem-st.27 & Gra-40924 & 5095 & 35 & Cereal (barley) \\
\hline Lumbres-La Montagne C VIII & Gif-5054 & 5100 & 80 & Charcoal \\
\hline Raillencourt-st55-19 & Ly1265 & 5110 & 55 & Charcoal \\
\hline Spiere-De Hel & KIA-15236 & 5170 & 35 & Hazelnut \\
\hline Corbehem-st.14 & GrA-38529 & 5205 & 35 & Hazelnut \\
\hline Corbehem-st.22 & GrA-38556 & 5210 & 45 & Cereal (wheat) \\
\hline Spiere-De Hel & KIA-15239 & 5255 & 35 & Hazelnut \\
\hline Corbehem-st.6 & GrA-38527 & 5280 & 35 & Charcoal \\
\hline Lumbres-La Montagne C IX & Gif-5055 & 5290 & 80 & Charcoal \\
\hline Lauwin-Planque-1437-11 & Ly1745 & 5290 & 45 & Charcoal \\
\hline Raillencourt-st55-7 & Ly1264 & 5295 & 55 & Charcoal \\
\hline Lauwin-Planque-1437-5 & Ly1744 & 5320 & 40 & Charcoal \\
\hline Kemmelberg & Lv-599 & 5430 & 100 & Charcoal \\
\hline Spiere-De Hel & Gr>N-22661 & 5620 & 80 & Charcoal \\
\hline
\end{tabular}

Tab. I - Datations ${ }^{14} \mathrm{C}$ des sites du Néolithique moyen II du Nord de la France et de la Belgique (hors minières).

\begin{tabular}{|l|l|r|r|l|c|}
\hline \multicolumn{1}{|c|}{ site } & Lab number & date BP & \multicolumn{1}{c|}{ stdev } & Material & VANMONTFORT 2004 \\
\hline Lumbres-La Montagne c B & Gif-? & 4368 & 130 & Charcoal & large standard deviation / association / charcoal \\
\hline Lumbres-La Montagne & Gsy-49 & 4470 & 200 & Charcoal & large standard deviation / association / charcoal \\
\hline Lumbres-La Montagne c B & Gif-? & 4571 & 130 & Charcoal & large standard deviation / association / charcoal \\
\hline Spiere-De Hel & GrN-22663 & 4890 & 70 & Charcoal & charcoal / other dates available \\
\hline Kemmelberg & Lv-524 & 5000 & 120 & Charcoal & large standard deviation / other date available / charcoal \\
\hline Liévin & Gif-4022 & 5170 & 120 & Charcoal & charcoal / large standard deviation \\
\hline Spiere-De Hel & GrN-22662 & 5400 & 40 & Charcoal & charcoal / other dates available \\
\hline Liévin & Gif-4023 & 5400 & 120 & Charcoal & charcoal / large standard deviation \\
\hline
\end{tabular}

Tab. II - Mesures radiocarbone écartées avant cet article (VANMONTFORT 2004).

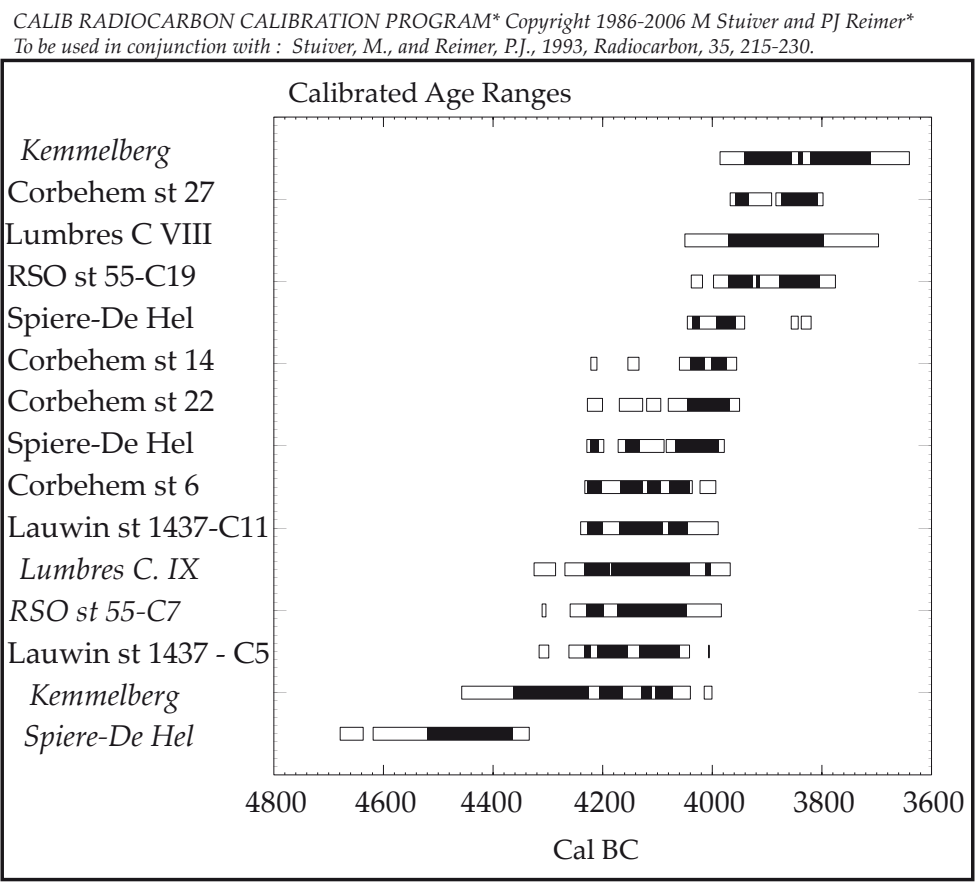

Fig. 9 - Classement des mesures radiocarbone (en italique les dates écartées). 
Rappelons que la précision d'une mesure radiocarbone varie selon la marge statistique de probabilité qui lui est accolée (OBERLIN 2003). Par exemple, pour ce qui nous concerne, une date de $5430 \mathrm{BP}+/-100$ promet à $95 \%$ de se ranger dans un intervalle de temps couvrant 460 ans en années réelles. À l'inverse, une date avec une marge statistique plus réduite, $5255 \mathrm{BP}+/$ - 35, permet de reconnaître l'évènement à dater dans un intervalle contraint sur deux siècles et demi. Plus la marge statistique est faible plus on réduit les plages d'incertitude. Mais, l'intervalle de temps en années réelles, quelle que soit la marge statistique, reste toujours important lorsque l'on se situe sur une période défavorable de la courbe de calibration.

Plusieurs échantillons sur charbons de bois, dont les essences n'ont pas été déterminées, peuvent être victimes de l'effet « vieux bois ». Dans ce cadre, les trois-quarts de l'échantillon doivent être utilisés avec précaution. La date provenant du comblement d'un des tronçons du fossé d'enceinte du site de Spiere (GrN-22661, tab. I) semble peu fiable car son caractère ancien la contraint à l'isolement et la désigne comme étant victime de l'effet « vieux bois », elle présente, en outre, une marge statistique importante (+/ - 80). L'analyse anthracologique des échantillons du site de Spiere "De Hel" avait montré que la plupart des charbons de bois provenant de cette tranchée était du chêne, essence plutôt sensible à l'effet «vieux bois »(VANMONTFORT et al. 2004). C'est pourquoi, deux autres dates (GrN-22662 et GrN 22663, tab. II), obtenues à partir de charbons de bois issus du même fossé d'enceinte, avaient déjà été écartées (VANMONTFORT 2004).

Les mesures obtenues sur le site de Kemmelberg (Lv-599 et Lv-525) situées aux deux extrémités de la courbe sont aussi discutables. La plus ancienne (Lv-599) est soupçonnée de recéler les biais liés aux mesures réalisées sur des charbons de bois, mais surtout ces deux mesures radiocarbones présentent une marge statistique élevée (+/ - 100 et 95 ans) qui, rajoutée aux incertitudes de la courbe de calibration, donne des résultats très dispersés ; ces deux datations seraient donc à écarter.

Les contextes de prélèvement semblent $a$ priori fiables puisque dans la plupart des cas ces échantillons sont associés à un mobilier archéologique homogène et récupérés au sein des comblements d'ensembles cohérents. Dans le détail, la situation est un peu différente.

À Lauwin-Planque "rue J. Cartier", les prélèvements ont été réalisés au sein des mêmes structures mais dans deux couches différentes (c. 5 et c. 11) qui appartiennent aux premières phases du remplissage et qui sont associées au dépôt des vases (Bostyn et al. 2006). Les résultats des datations sont très proches (fig. 9) et malgré l'antériorité de l'échantillon de la couche 5 par rapport à celui provenant de la couche 11 qui lui est sous-jacente, la superposition des résultats à 1 sigma témoignerait en faveur d'une phase de comblement relativement rapide du fossé principal de l'enceinte (fig. 10).

À Raillencourt-Sainte-Olle, la dynamique de comblement de la structure 55 (un puits) fait se succéder les couches 19 et 7 et le temps les séparant paraît très court. Les charbons de bois ont été prélevés au sein de ces couches. Cette succession rapide pourrait expliquer l'ancienneté de la couche 7 par rapport à la couche 19 alors que la stratigraphie indique l'inverse. Mais ici, l'écart entre ces deux mesures est tellement important qu'elles s'excluent l'une et l'autre à 1 comme à 2 sigma (fig. 9 et 10). L'effet "vieux bois » peut être avancé pour le résultat le plus ancien, ce qui nous conduirait alors à ne conserver que la mesure la plus récente (c 19) qui fait partie d'une des cinq dates situées en dehors d'un des phénomènes de plateau reconnus sur la courbe de calibration.

Sur les deux mesures du site de Lumbres, seule celle provenant de la couche VIII doit être conservée car elle est associée avec assurance à un mobilier attribué dans un premier temps au Michelsberg (PININGRE 1980) avant d'être intégré au groupe de Spiere (VANMONTFORT 2004). La couche IX, sousjacente à la précédente, a livré un mobilier lithique (amas) avec des tessons attribuables au Cerny, peutêtre en position remaniée et doit être écartée.

En considérant l'ensemble de ces critiques, plusieurs dates réunissent trop d'incertitudes sur leur provenance contextuelle, sur leurs marges statistiques, sur la nature du matériau daté. Le cumul de ces éléments constitue, pour nous, un motif suffisant pour les laisser de côté.

\section{CHRONOLOGIE DU GROUPE DE SPIERE}

Après ce premier tri, dix dates ont été conservées. Leurs mesures radiocarbones se répartissent sur quatre siècles environ situés en années réelles entre 4260 et 3810 ans (fig. 10). Les dates les plus anciennes concernent les enceintes de Lauwin-Planque et de Spiere, avec un terminus situé au cours du $40^{\mathrm{e}}$ siècle avant notre ère. Les sites à fosse recouvrent quant à eux la totalité de la durée d'existence du groupe de Spiere, mais avec la moitié des dates (3 sur 6) centrée sur la fin du $39^{\text {e }}$ siècle avant notre ère et qui concerne les sites de Corbehem, de Lumbres (c. VIII) et de Raillencourt-Sainte-Olle (c. 19). Le gisement de Corbehem "rue de Gouy" donne des résultats recoupant la totalité des quatre siècles d'existence, avec à chaque fois une date par fosse laissant envisager une fréquentation régulière de ces populations. La superposition des types d'occupation et leur succession dans le temps demandent à être affinées par de nouveaux résultats 
de datation mais surtout demandent à être étayées par les études typo-technologiques des mobiliers archéologiques. Dans l'attente de nouvelles mesures radiocarbone et découvertes, il est intéressant de noter que l'émergence des enceintes dans la région se manifeste dès les débuts du Néolithique moyen II et que seules deux structures du site de Corbehem (st 6 et 14 qui n'ont pas livré de mobilier datant) leurs seraient contemporaines. Ensuite, dans la seconde partie de la séquence, la plus récente, il ne semble persister dans la région que des sites à fosses. Les deux enceintes datées, Lauwin-Planque et Spiere, n'ont de toute façon pas livré de datations plus récentes. Pour Spiere au moins ceci semble significatif car les deux mesures disponibles datent en effet la fin du remplissage du fossé.

\section{CONCLUSION}

$\mathrm{Au}$ cours des dix dernières années, plusieurs gisements situés entre la fin du Ve et le début du IV ${ }^{\mathrm{e}}$ millénaires avant notre ère, ont été fouillés dans la région Nord - Pas-de-Calais. Même si tous les corpus de mobilier ne sont pas encore exhaustivement

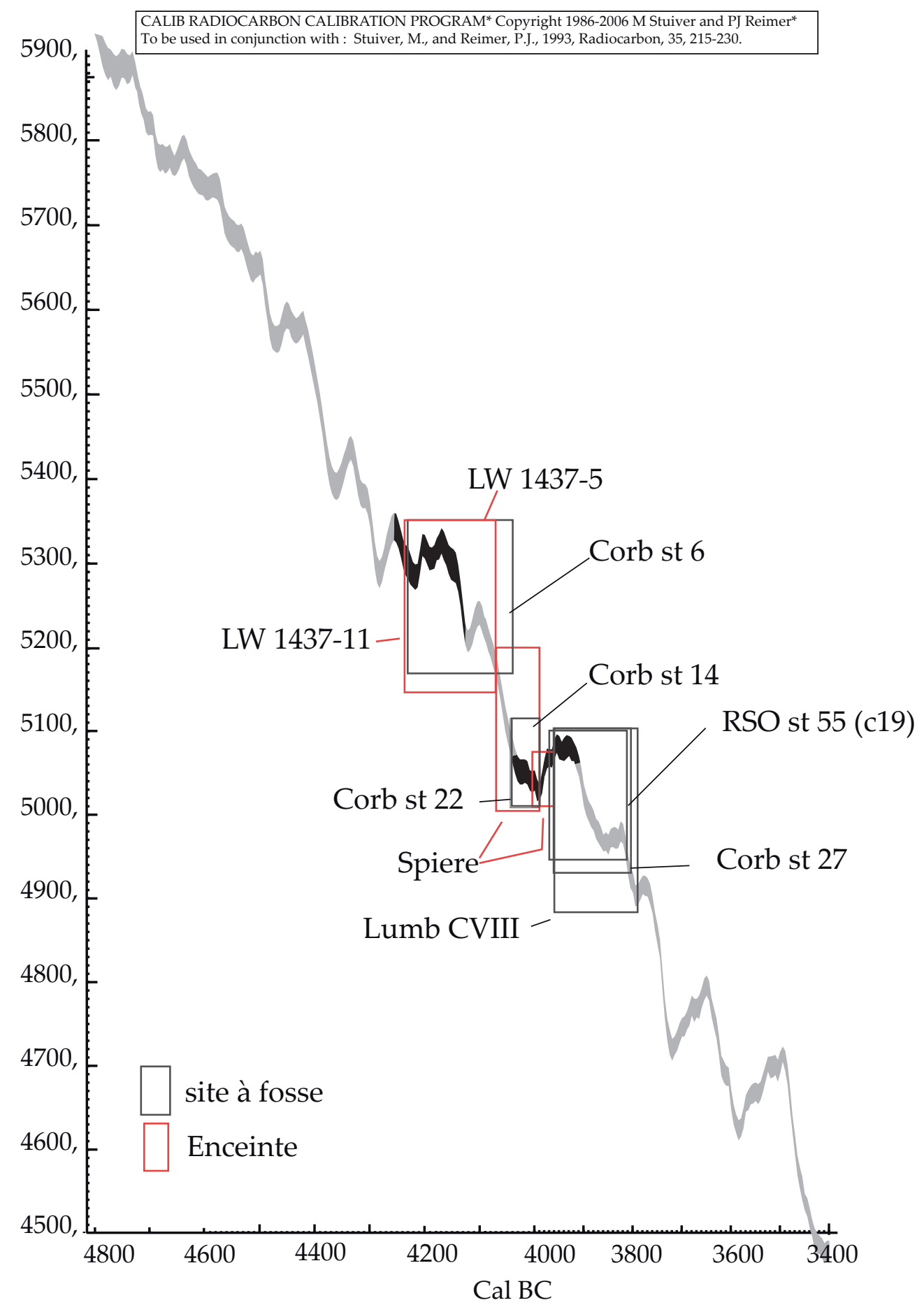

Fig. 10 - Résultat des mesures radiocarbone à 1 sigma (rectangle) projeté sur la courbe de calibration des sites à fosses et des enceintes du groupe de Spiere (en noir sur la courbe nous avons matérialisé les «âges plateaux »). 
étudiés, ces nouvelles données montrent que les caractères originaux qui avaient permis de définir le groupe de Spiere sont représentés sur les sites du Nord de la France tant dans le mobilier céramique que dans l'industrie lithique. L'extension géographique et culturelle du groupe de Spiere est confortée ; elle se trouve fortement ancrée dans le sud-ouest du Bassin de l'Escaut (fig. 11). Vers l'est, elle est atténuée par l'existence du Michelsberg belge dont les contacts avec le groupe de Spiere sont indéniables (circulation de biens manufacturés, de matériaux...). On comprendra que les relations soient plus fortes avec ces sites, soulignées notamment par l'importation de lames en silex de Spiennes. Les limites «territoriales » à l'ouest et au sud sont beaucoup plus flottantes et ouvertes. Vers les côtes de la Manche / Mer du Nord, le nombre de découvertes récentes est plus restreint et les premiers éléments céramiques comme ceux réunis à Coquelles ou à Isques ne montrent pas de réelles différences avec ce qui est reconnu dans le reste de la région. On aurait pu s'attendre à la présence d'éléments plus marqués dans les assemblages mobiliers par des influences chasséennes ou Michelsberg. Mais les corpus sont encore trop faibles pour se prononcer : espérons que la fouille du site d'Escalles "Le Mont d'Hubert" pourra apporter des données précises et alimenter le débat sur les aires d'extension respectives des différents groupes. De la même manière, les découvertes récentes faites dans le Bassin de la Somme, devraient permettre de redéfinir les contours du groupe de Spiere au sud et de préciser la nature et l'intensité des contacts entretenus avec les gisements situés au nord du Bassin parisien.

Les premières analyses des séries céramiques et lithiques ne manifestent pas une évolution évidente au cours des quatre à cinq siècles d'existence du groupe de Spiere, suggérant au contraire une relative stabilité sur toute la période. Pourtant, les dates radiocarbones décrivent deux séquences principales au sein desquelles les premières grandes manifestations du Néolithique moyen II régional se traduisent par l'érection d'enceintes à fossés interrompus sur une durée de deux siècles tandis que les gisements ne livrant que des fosses auraient une existence étalée sur les quatre siècles du groupe de Spiere, mais principalement regroupés dans le premier quart du $\mathrm{IV}^{\mathrm{e}}$ millénaire avant notre ère. La prudence s'impose mais il faudra examiner en parallèle les séries provenant de sites dont la nature est par essence différente. Ce bloc apparemment sans nuances pourrait se révéler plus complexe dans son développement. Pour l'heure, le matériel céramique et lithique présente des caractéristiques

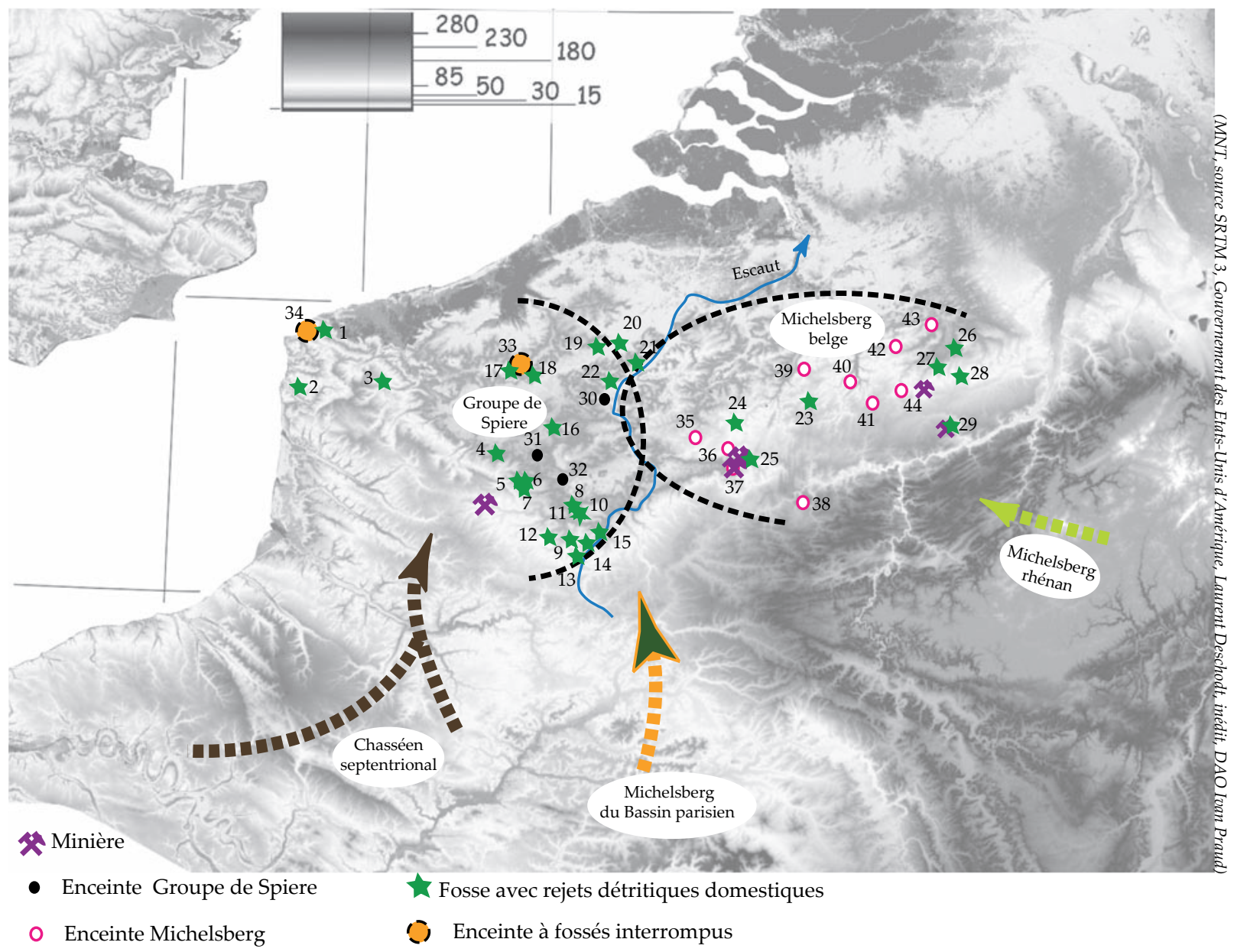

Fig. 11 - Répartition des sites dans l'aire d'influence du Groupe de Spiere et du Michelsberg Belge. 
typologiques et technologiques empruntées pour partie aux deux grandes entités culturelles voisines, le Michelsberg et le Chasséen septentrional dans leurs phases plutôt anciennes. Plusieurs éléments décorés montrent l'ascendance qu'a pu avoir le groupe de Menneville/Bischheim occidental dans la formation du groupe de Spiere. Là encore, il faut remarquer le caractère pluriel servant de fondement à l'existence de ce groupe culturel avec des éléments Épi-Roessen relevant d'influences plus orientales notamment dans le mobilier décoré de Sailly-laBourse.

Ce groupe régional porte en lui, dans sa genèse et son développement, le fait de se retrouver systématiquement au carrefour de deux grandes sphères d'influences produisant les sociétés néolithiques.

La possibilité d'une disparition des enceintes à la fin du Ve millénaire évoquée à titre d'hypothèse et qui devra être confortée par les recherches futures, montre que la définition du groupe de Spiere reste encore à préciser en faisant intervenir d'autres critères que les traditionnels corpus céramique, comme la nature et la fonction des sites.

\section{BIBLIOGRAPHIE}

BLANCHET Jean-Claude \& MARTINEZ Roger (1980) - "Vers une chronologie interne du Chasséen dans le Nord du Bassin parisien » dans DEMOULE Jean-Paul \& GUILAINE Jean (dir) - Le Néolithique de la France. Hommage à Gérard Bailloud, Paris, p. 331-342.

BLANCHET Jean-Claude \& BURNEZ Laurence (1984) - " Le camp chasséen du "Mont d'Huette" à Jonquières (Oise). La céramique décorée ", Revue archéologique de Picardie, 1/2, p. 217-242.

BLANCHET Jean-Claude, BOUCHAIN P., \& DECORMEILlE A. (1984) - " Le "Camp de César" à Catenoy (Oise). Bilan des anciennes recherches et des fouilles récentes de 1982 à 1983 » dans Colloque interrégional sur le Néolithique, Compiègne, sept. 1982, Revue archéologique de Picardie, 1/2, p. 173-204.

BOSTYN Françoise avec la collaboration d'Ivan PRAUD (1999) - Raillencourt-Sainte-Olle "Le Grand Camp", Rapport de fouilles préventives, SRA du Nord-Pas-de-Calais, 21 pages rexte, 42 fig.

BOSTYN Françoise, DEMOLON Pierre, FRANGIN Elsa \& Christian SEVERIN (2006) - " Un site d'habitat du Néolithique moyen II à Lauwin-Planque (Nord) : premiers résultats » dans Actes du colloque interrégional sur le Néolithique, Dijon, octobre 2001, Revue archéologique de l'Est, 25e supplément, p. 319-334.

BOSTYN Françoise, GAILLARD Denis, GUSTIAUX Michelle \& PRAUD Ivan (2006) - « Masnières. Allée des Tilleuls, la Couture », Bilan Scientifique Régional, SRA du Nord-Pas-de-Calais, p. 116-117.

BOSTYN Françoise, BOSSUT Dominique, DIESTCHSELLAMI Marie-France, DUFRAISSE Alexa, FAUPIN Géraldine, FAVIER Dominique, LANCELOT Stéphane,
MONCHABLON Cécile, PRAUD Ivan \& WATTEZ Julia (2009) - Corbehem "Rue de Gouy" (Pas-de-Calais), INRAP, SRA Nord-Pas-de-Calais.

CONSTANTIN Claude \& DEMAREZ Léonce (2009) "L'enceinte Michelsberg de Blicquy "La Couture du Couvent" », Anthropologica et Praehistorica, 120, p. 151202.

CONSTANTIN Claude \& KUIJPER Wim. J. (2002) "Utilisation de mousse comme dégraissant dans des céramiques néolithiques de France et de Belgique ", Bulletin de la Société Préhistorique Française, 99 (4), p. 775-784.

DE HEINZELIN Jean, HAESAERTS Paul \& DE LAET Sigfried J. avec la collaboration de BASTIN Bruno, CZEPIEC Stanislaw, GAUTIER Achilles, HULTEN Birgitta, SPLINGAER Marcel \& VAN DOORSELAER André (1977) - Le Gué du Plantin (Neufvilles, Hainaut), site néolithique et romain, Bruges, (Dissertationes Archaeologicae Gandenses, 17), 146 p.

DEBIAK Rudy \& DEBS Ludovic (1996) - «Beaulencourt / Arleux-en-Gohelle (gazoduc)», Bilan scientifique Régional. SRA du Nord-Pas-de-Calais, p. 70.

DECHEYER Nathalie (2006) - « Coquelles "Les Terrasses de Coquelles" (Pas-de-Calais) », Bilan Scientifique Régional, SRA du Nord-Pas-de-Calais, 2000, p. 218

DEMOLON Pierre \& HURTRELLE Jacques (1975) "Le site néolithique de Corbehem (Pas-de-Calais). Considérations sur le "groupe Michelsberg Belge" », Septentrion, 5, p. 40-44.

DUBOULOZ Jérôme (1988) - Le style de Menneville et les débuts du Chalcolithique dans la France $d u$ Nord, Thèse de doctorat Paris, Université de Paris I.

DUBOULOZ Jérôme (1991) - « Le village fortifié de Berryau-Bac et la signification pour la fin du Néolithique dans la France du Nord » dans LICHARDUS Jan (éd). - Die Kupferzeit als historische Epoche, (Saarbrücker Beiträge zur Alterumskunde 55) Bonn, p. 421-440.

DUBOULOZ Jérôme (1998) - « Réflexions sur le Michelsberg ancien en Bassin parisien » dans BIEL Jörg, SCHLICHTHERLE Helmut, STROBEL Michael \& ZEEB Andrea (eds) - Die Michelsberger Kultur und ihre Randgebiete. Probleme der Entstehung, Chronologie und des Siedlungswesens, (Materialhefte zur Archäologie in Baden-Württemberg 43), Stuttgart, p. 9-20.

DUBOULOZ Jérôme, LASSERRE Marina \& LEBOLLOCH Marie-Annick (1984) - «Éléments pour une chronologie relative des ensembles Roessen, Post-Roessen, Michelsberg et Chasséen dans la vallée de l'Aisne et le Bassin parisien » dans Actes du $9^{\mathrm{e}}$ colloque interrégional sur le Néolithique (Compiègne 1982). Revue archéologique de Picardie, 1/2, p. 111-123.

FERAY Plilippe (2003) - « Avion. Échangeur RN17. ZAC de la Glissoire, bassin nord ", Bilan Scientifique Régional, SRA du Nord-Pas-de-Calais, p. 111-114.

GAILLARD Denis \& GUSTIAUX Michèle (2009) - Rapport de diagnostic ZD 8 Oisy-le-Verger et Sauchy-Lestrée. Canal Seine-Nord Europe, Service Régional du Nord - Pas-deCalais, Lille, 118 p, 2 annexes, 3 inventaires.

GLESER Ralph (1995) - Die Epi-Rössener Gruppen in Südwestdeutschland. Untersuchungen zur Chronologie, stilistischen Entwicklung und kulturellen Einordnung, 
(Saarbrücker Beiträge zur Alterumskunde ; 61) Bonn, 408 p., $66 \mathrm{pl}$.

HAMON Caroline (2006) - Broyage et abrasion au Néolithique ancien. Caractérisation technique et fonctionnelle de l'outillage en grès du Bassin parisien (BAR International Series 1551), Oxford.

JACQUES Alain, HOSDEZ Christophe \& ROSSIGNOL P.atrick (1995) - « Le Néolithique moyen à Monchy-lePreux ", Histoire et Archéologie du Pas-de-Calais, XIV, n 1, p. $1-8$.

JEUNESSE Christian (1982) - « Les influences épi-roessen et Michelsberg dans le nord-est du Bassin parisien et en Belgique occidentale : analyse chronologique ", Revue archéologique de Picardie, 4, p. 49-65.

JEUNESSE Christian (1998) - « Pour une origine occidentale de la culture de Michelsberg ? » dans BIEL Jörg, SCHLICHTHERLE Helmut, STROBEL Michael \& ZEEB Andrea (eds) - Die Michelsberger Kultur und ihre Randgebiete. Probleme der Entstehung, Chronologie und des Siedlungswesens, (Materialhefte zur Archäologie in Baden-Württemberg 43), Stuttgart, p. 29-46.

JEUNESSE, Christian, LEFRANC Philippe \& DENAIRE Anthony avec les contributions de ARBOGAST RoseMarie \& NAZE Gilles (2004) - Groupe de Bischheim, origine du Michelsberg, genèse du groupe d'Entzheim. La transition entre le Néolithique moyen et le Néolithique récent dans les régions rhénanes, (Cahiers de l'Association pour la Promotion de la Recherche Archéologique en Alsace 18/19, (2002/2003)), Zimmersheim.

LANCHON Yves (dir.), ARNOLD B., AUGEREAU Anne, BADALIAN L., BINET Christian, BOGUSZEWSKIAndrej, BULARD Alain , BURET Gérard, CHAMBON Philippe, DELATTRE Nathalie, DIETSCH Marie-France, DIQUERO Gwenaelle, DURAND Stéphane, ERNST Thomas, EVIN Jacques, FONTUGNE M., FRUGIER Catherine, LAFAGE Françoise, LEROYER Chantal, LIMONDIN Nicole, MARQUIS Philippe, MARTINEZ Roger, MAZURIE DE KEROUALIN François, MONCHABLON Cécile, ROBLIN-JOUVE Annie, PERNAUD Jean-Marie, RAMSEYER Denis, RICQ de BOUARD Monique, RODRIGUEZ Patrice, SALANOVA Laure, TRESSET Anne, VALERO Carlos \& WATTEZ Julia (1998) - Le cadre naturel et les occupations néolithiques du site de Paris Bercy, DFS 1998, SRA Île-de-France, 5 volumes

LANTOINE Jennifer, BOSTYN Françoise \& HENTON Alain (2006) - « Courrières. Rues Casimir Beugnet et Pierre Bouchez », Bilan Scientifique Régional, SRA du Nord-Pasde-Calais, p. 219.

LE BOLLOCH Mariannick (1984) - « Le Michelsberg dans la vallée de l'Aisne. Le Néolithique dans le Nord de la France et le Bassin parisien » dans Actes du Colloque interrégional sur le Néolithique. Compiègne les 24, 25, 26 septembre 1982, Revue archéologique de Picardie, 1/2, p. 133-146.

LEMAN Pierre (1982) - « Brebières », Informations, GalliaPréhistoire, 25 (2), p. 244.

LEMAN Pierre (1978) - « Sailly-Labourse », Informations, Gallia-Préhistoire, 21 (2), p. 467.

LEMAN Pierre \& LEMAN Germaine (1973) - « Une fosse du Néolithique moyen à Estrun (Nord) », Revue du Nord, 55, p. 7-15.
LEPAUMIER Hubert (1996) - «Isques », Bilan Scientifique Régional, 1995, SRA du Nord-Pas-de-Calais, p. 99-101.

LICHARDUS Jan (1976) - Rössen - Gatersleben - Baalberge. Ein Beitrag zur Chronologie des mitteldeutschen Neolithikums und zur Entstehung der Trichterbecherkulturen, (Saarbrücker Beiträge zur Altertumskunde 17), Bonn.

LOMBARDO Jean-Luc, MARTINEZ Roger \& VERRET Daniel (1984) -« Le site Chasséen du "Cul-Froid" à Bouryen-Vexin dans son contexte historique et les apports de la stratigraphie de son fossé » dans Le Néolithique dans le Nord de la France et le Bassin parisien. Actes du $9^{\mathrm{e}}$ colloque interrégional sur le Néolithique, Compiègne 1982, Revue archéologique de Picardie, 1/2, p. 269-284.

LOUWE KOOIJMANS Leendert P. (1976) - « Local Developments in a Borderland. A Survey of the Neolithic at the Lower Rhine ", Oudheidkundige Mededelingen uit het Rijksmuseum van Oudheden te Leiden, 57, p. 227-298.

LOUWE KOOIJMANS Leendert P. (1980) - « De middenneolithische vondstgroep van het Vormer bij Wijchen en het cultuurpatroon rond de Zuidelijke Noordzee circa 3000v.Chr. ", Oudheidkundige Mededelingen van het Rijksmuseum van oudheden te Leiden, 61, p. 113-208.

LÜNING Jens (1968) - «Die Michelsberg Kultur. Ihre Funde in zeitlicher und räumlicher Gliederung », Bericht der römisch-germanischen Kommision, 48, p. 1-350.

MANCEAU Lorraine (ce volume) - « La céramique Néolithique moyen du site de Lauwin-Planque "J. Cartier" » (Nord) : étude typo-technologique ».

MASSON Bertrand \& VALLIN Luc (1994) - « Un site d'extraction de silex à Fampoux "Le Réservoir" (Pas-deCalais) », Les Cahiers de Préhistoire du Nord/Pas-de-Calais, 16, p. 7-40.

MONCHABLON Cécile (1999) - « Le matériel en grès et calcaire » dans HACHEM Lamys (dir.) - Bucy-le-Long "La Fosselle" (Aisne), Rapport de Fouille 1997, SRA de Picardie, Amiens,1999.

MONCHABLON Cécile (2005) - «Le matériel de mouture, de broyage et de polissage » dans GILIGNY Fr. (dir.) - Louviers "La Villette" (Eure) : Un site néolithique moyen en zone humide, Rennes, Documents Archéologiques de l'Ouest, p. 149- 161

MONCHABLON Cécile, BAILLIEU Michel, BOUCHET Marie, GOUTELARD Arnaud \& PRAUD Ivan (ce volume) - « L'enceinte néolithique de Carvin "La Gare d'Eau" (Pasde-Calais) ».

OBERLIN Christine (2003) - « Calibration des datations radiocarbone : le point sur la période $\mathrm{VI}^{\mathrm{e}}-\mathrm{II}^{\mathrm{e}}$ millénaire avant J.-C. » dans GASCO, GUTHERZ \& LABRIFFE (dir.) - Temps et espaces culturels du VI au II millénaire en France du Sud, actes des $4^{\mathrm{e}}$ Rencontres Méridionales de Préhistoire Récente, Nîmes 28 et 29 oct. 2000, (Monographies d'archéologie méditerranéenne 15), Lattes, p. 35-42

PININGRE Jean-François (1980) - « La stratigraphie du site néolithique de la "Montagne de Lumbres". État des recherches, 1977-78 », Préhistoire et Protohistoire en Champagne-Ardennes, n spécial, p. 109-117.

PININGRE Jean-François (1985) - « Le site néolithique moyen de Liévin (Pas-de-Calais) », Bulletin de la Société Préhistorique Française, 82, p. 422-448 
PIRSON Stéphane DUPUIS Christian, BAELE Jean-Marc, COLLET Hélène \& MORTIER Thierry (2001) - « Fragments de polissoirs découverts à Petit-Spiennes : pétrographie et implications archéologiques », Notae Praehistoricae, 21, p.145-156

PREVOST Dom R. (1959) - "Fosse d'extraction de silex à Lumbres (Pas-de-Calais) ", Bulletin de la Société Préhistorique Française, 3/4, p. 161-162.

PREVOST Dom R. (1962) - L'habitat néolithique de la "Montagne de Lumbres", (Mémoires de la Commission Départementale des Monuments Historiques du Pas-deCalais XI), Arras, 95 p.

SCOLLAR Irwin (1959) - " Regional groups in the Michelsberg culture : a study in the Middle Neolithic of west central Europe », Proceedings of the Prehistoric Society, 25 , p. 52-134.

TUFFREAU Alain (1970) - « La fosse chasséenne de Liévin (Pas-de-Calais) », Bulletin de la Société Préhistorique Française, 67 (2), p. 43-46.

VANDOORSELAER (1971) - «Inlendendebeschouwingen over de Kemmelberg na drie opgravingscampagnes », Archaeologia Belgica, 131.

VAN DOORSELAER André, PUTMAN Robert, VAN DER GUCHT K. \& JANSSENS F. (1987) - De Kemmelberg, een Keltische bergvesting. Voorstelling van het aarden vaatwerk, (Westvlaamse Archaeologica Monografieën 3), Kortrijk

VANMONTFORT Bart (2001) - « The group of Spiere as a new stylistic entity in the middle Neolithic Scheldt basin », Notae Praehistoricae, 21, p. 139-143.

VANMONTFORT Bart (2004) - Converging worlds. The neolithisation of the scheld t basin during the late fifth and early fourth millennium cal BC. Thèse de doctorat. Katholieke Universiteit Leuven.

VANMONTFORT Bart (2005) - « Techno-functional aspects of a middle Neolithic pottery assemblage (Spiere "De Hel", Belgium) » dans LIVINGSTONE-SMITH Alexandre, BOSQUET Dominique \& MARTINEAU Rémi (eds) - Pottery Manufacturing Processes : Reconstruction and Interpretation, (BAR International Series 1349), Oxford, p. $115-125$.
VANMONTFORT Bart (2006) - «Can we attribute the Middle Neolithic in the Scheldt and Middle Meuse basins to the Michelsberg Culture? » dans DUHAMEL Pascal (ed.) - Impacts interculturels au Néolithique moyen. Du terroir au territoire: sociétés et espaces, (Revue Archéologique de l'Est. Suppléments 25), Dijon, p. 109-116.

VANMONTFORT Bart, CASSEYAS Christian, VERMEERSCH Pierre M. (1997) - « Neolithic ceramics from Spiere "De $\mathrm{Hel}^{\prime}$ and their contribution to the understanding of the earliest Michelsbergculture », Notae Praehistoricae, 17, p. 123-134.

VANMONFORT Bart, GEERTS Anne-Isabelle, CASSEYAS Christian, BAKELS Corrie, BUYDENS Christophe, DAMBLON Freddy, LANGHOR Roger, NEER Wim Van \& VERMEERSCH Pierre M. (2004) - « De Hel in de tweede helft van het 5 de millennium v.Chr. Een middenNeolithische enclosure te Spiere (prov. West-Vlaanderen) », Archeologie in Vlaanderen, VIII (2001/2002), p. 9-77.

VANMONTFORT Bart, COLLET Hélène \& CROMBÉ Philippe (2009) - «Les industries lithiques taillées des IVe et III $^{\mathrm{e}}$ millénaires dans les bassins de l'Escaut et de la Meuse (Belgique) » dans DIAS-MEIRINHO M.-H., LÉA V., GERNIGON K., FOUÉRÉ, P., BRIOIS F. \& BAILLY, M. (eds) - Les industries lithiques taillées des IVeet III millénaires en Europe occidentale, (BAR International Series 1884), Oxford, p. 11-39.

VERMEERSCH Pierre M. \& WALTER René (1978) Thieusies "ferme de l'Hosté", site Michelsberg, I, (Achaeologia Belgica 230), Bruxelles.

VERMEERSCH Pierre M., VYNCKIER Geert \& WALTER René (1990) - Thieusies, ferme de l'Hosté, site Michelsberg. IILe matériel lithique, (Studia Praehistorica Belgica 6).

VERMEERSCH Pierre M. \& BURNEZ-LANOTTE Laurence (1998) - «La culture de Michelsberg en Belgique, état de la question » dans BIEL Jörg, SCHLICHTHERLE Helmut, STROBEL Michael \& ZEEB Andrea (eds) Die Michelsberger Kultur und ihre Randgebiete. Probleme der Entstehung, Chronologie und des Siedlungswesens, (Materialhefte zur Archäologie in Baden-Württemberg 43), Stuttgart, p. 47-54.

WILLOT Jean-Michel (2007) - « Escalles "Mont d'Hubert" », Bilan Scientifique Régional, Nord-Pas-de-Calais, p. 175-179. 


\title{
Les auteurs
}

\author{
Françoise BOSTYN \\ Inrap \& UMR 7041, Protohistoire européenne \\ 36 Allée Thalès \\ 59650 Villeneuve d'Ascq \\ francoise.bostyn@inrap.fr \\ Cécile MONCHABLON \\ Inrap \& UMR 7041, Protohistoire européenne \\ Centre archéologique Inrap \\ 32 rue Delizy \\ 93500 Pantin \\ cecile.monchablon@inrap.fr \\ Ivan PRAUD \\ Inrap \& UMR 7041, Protohistoire européenne \\ Centre archéologique Inrap \\ 11 rue des Champs \\ 59650 Villeneuve d'Ascq \\ ivan.praud@inrap.fr

\section{Bart VANMONTFORT} \\ CNRS, Protohistoire européenne UMR 7041 \& \\ Prehistoric Archaeology Unit, Katholieke Universiteit Leuven \\ Celentijnenlaan 200E pb 2409 \\ 3001 Leuven \\ Belgique \\ bart.vanmontfort@ees.kuleuven.be
}

\section{Résumé}

Depuis une décennie, la documentation concernant le Néolithique moyen II du Nord de la France s'est renouvelée et étoffée notamment grâce à l'archéologie préventive. Ces nouvelles données montrent que les caractères originaux qui avaient permis de définir le groupe de Spiere sont représentés sur les sites du Nord de la France tant dans le mobilier céramique que dans l'industrie lithique. Les premières analyses des séries céramiques et lithiques ne manifestent pas une évolution évidente au cours des quatre ou cinq siècles d'existence de ce groupe, suggérant au contraire une relative stabilité sur toute la période. Pourtant, les dates radiocarbone décrivent deux séquences principales avant et après 4000 avant J.-C., encore difficiles à expliquer, mais qui pourraient recouper la nature ou la fonction différentes des sites. En tout état de cause, ces nouvelles données confirment l'impact des deux grandes entités culturelles voisines, le Michelsberg et le Chasséen septentrional, au sein de la culture matérielle.

Mots-clefs : Spiere, Nord de la France, céramique, datations radiocarbones

\begin{abstract}
During the last decade, developer-funded preventive archaeology has enlarged the information with regard to the Middle Neolithic II in the north of France. These new data show the presence of characteristics, both with respect to pottery and lithic technology, that allowed the identification of the group of Spiere. This group persisted during a period of 4 to 5 centuries. To date, no clear internal evolution can be observed in the pottery and lithic assemblages. Nevertheless a chronological subdivision, largely before and after $4000 \mathrm{cal}$ BC, can be proposed as a working hypothesis on the basis of the nature or function of the sites. In any case, the new data confirm the importance of both the northern Chasseen and the Michelsberg culture in the formation of the Spiere group.
\end{abstract}

Keywords : Spiere, The North of France, ceramic, radiocarbon dates 


\section{Zusammenfassung}

In den zehn Jahren wurde die Dokumentation zum Mittelneolithikum II in Nordfrankreich insbesondere Dank der Präventivarchäologie bereichert und ergänzt. Die neuen Ergebnisse zeigen, dass die Eigenschaften, die es ermöglicht hatten, die Spiere-Gruppe zu definieren, auf den Fundstätten Nordfrankreichs sowohl im Bereich der Keramik als auch bei den Steinwerkzeugen vertreten sind. Die ersten Analysen dieser beiden Fundkategorien lassen in den vier oder fünf Jahrhunderten, in denen die Spiere-Gruppe existierte, keine offensichtliche Entwicklung erkennen, sie legen im Gegenteil eine relative Stabilität während der ganze Periode nahe. An den Radiokarbondaten sind vor und nach 4000 v. Chr. allerdings zwei Hauptsequenzen erkennbar. Zwar sind diese noch schwer zu erklären, sie könnten jedoch einer unterschiedlichen Natur oder Funktion der Plätze entsprechen. Auf jeden Fall bestätigen diese neuen Daten den Einfluss der beiden großen kulturellen Nachbargruppen, der Michelsberger Kultur und dem Chasséen septentrional, auf die materiellen Kultur.

Schlagwörter : Spiere, Nordfrankreich, Keramik, Radionkarbondatierungen.

Traduction : Isa ODENHARDT-DONVEZ (donvezservit@wanadoo.fr). 
